\title{
Plant Specialized Metabolites in Hazelnut (Corylus avellana) Kernel and Byproducts: An Update on Chemistry, Biological Activity, and Analytical Aspects'
}

Authors

Alfredo Bottone, Antonietta Cerulli, Gilda D’Urso, Milena Masullo, Paola Montoro, Assunta Napolitano, Sonia Piacente

\section{Affiliation}

Dipartimento di Farmacia, Università degli Studi di Salerno, Fisciano (SA), Italy

Key words

Corylus avellana, Betulaceae, phenolics, diarylheptanoids, taxanes, biological activities, analytical tools

received February 14, 2019

revised May 28, 2019

accepted June 2, 2019

Bibliography

DOI https://doi.org/10.1055/a-0947-5725

Published online June 27, 2019 | Planta Med 2019; 85: 840-

855 (c) Georg Thieme Verlag KG Stuttgart · New York I

ISSN 0032-0943

Correspondence

Prof. Sonia Piacente

Dipartimento di Farmacia, Università degli Studi di Salerno

Via Giovanni Paolo II 132, 84084 Fisciano (SA), Italy

Phone: + 39089969763 , Fax: + 39089969602

piacente@unisa.it

\section{ABSTRACT}

Corylus avellana (hazelnut) is one of the most popular tree nuts on a worldwide basis. The main products of $C$. avellana are kernels, a nutritious food, with a high content of healthy lipids, contained in a hard shell. In recent years, along with the ongoing research carried out on hazelnut kernels, a growing interest has been addressed to the hazelnut byproducts including hazelnut skin, hazelnut hard shell, and hazelnut green leafy cover as well as hazelnut tree leaf. These byproducts deriving from the roasting, cracking, shelling/hulling, and harvesting processes have been found as a source of "phytochemicals" with biological activity. The aim of this review is to provide a comprehensive and critical update on the chemistry and biological activity of specialized metabolites occurring in hazelnut kernels and byproducts. Phenolics are the most abundant phytochemicals not only in the kernels, but also in other processing byproducts. Attention has been also devoted to taxane derivatives isolated from C. avellana leaves. An overview on the biological activity, mainly antioxidant, antiproliferative, and antimicrobial along with less common biological effects, has been provided, contributing to highlight $C$. avellana as a source of bioactive phytochemicals with the potential to exert beneficial effects on human health. Finally, analytical techniques for the quali-quantitative analysis of specialized metabolites occurring in the different parts of $C$. avellana have been reviewed.

$\begin{array}{ll}\text { ABBREVIATIONS } \\ \text { AGE } & \text { advanced glycation end-product } \\ \text { DAD } & \text { diode-array detector } \\ \text { DPPH } & \text { 1,1-diphenyl-2-picrylhydrazyl } \\ \text { MIC } & \text { minimal inhibitory concentration } \\ \text { SPE } & \text { solid-phase extraction } \\ \text { TBARS } & \text { thiobarbituric acid reactive substances } \\ \text { TDDFT } & \text { time-dependent density functional theory } \\ \text { TEAC } & \text { trolox equivalent antioxidant capacity } \\ \text { UA-SLE } & \text { ultrasound-assisted solid/liquid extraction }\end{array}$

\section{Introduction}

Corylus avellana L. (Betulaceae) is one of the most popular tree nuts on a worldwide basis. The main products of $C$. avellana are kernels, a nutritious food with a high content of healthy lipids [1], used by the confectionary industry and consumed raw (with skin) or preferably roasted (without skin). The kernel is contained in a hard shell. Hazelnut skin, hazelnut hard shell, and hazelnut green leafy cover as well as hazelnut tree leaf are byproducts of roasting, cracking, shelling/hulling, and harvesting processes, respectively. Food plant-derived products contain, along with pri- 

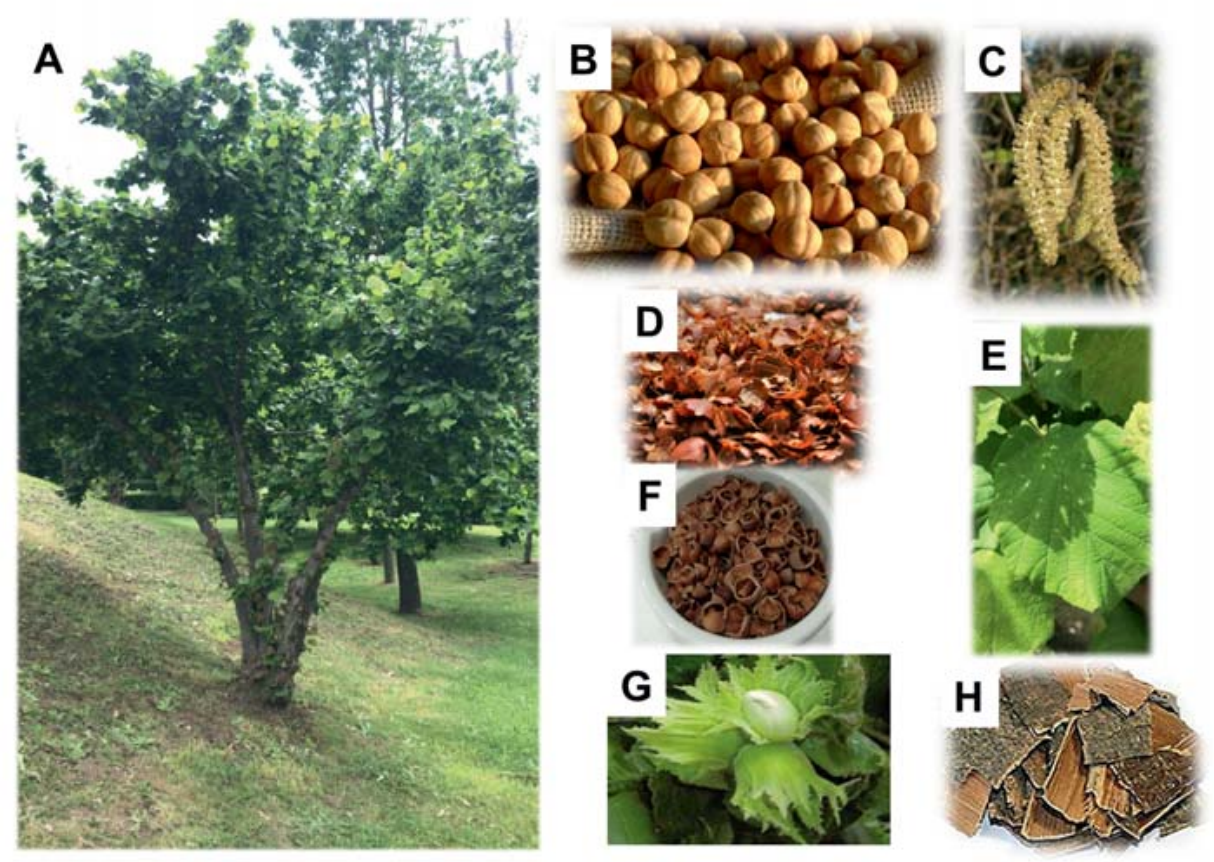

- Fig. 1 C. avellana: (A) tree, (B) nuts, (C) male flowers, (D) skins, (E) leaves, (F) shells, (G) leafy covers, (H) bark.

mary constituents, a wide range of specialized metabolites, well known as "phytochemicals," that possess substantial biological activity. In recent years, together with the ongoing research carried out on hazelnut kernels, a growing interest has been addressed to the hazelnut byproducts.

Among hazelnut phytochemicals, phenolic compounds represent the main specialized metabolites and are of considerable interest due to their biological activity [2-4]. Moreover, the finding of taxanes in in vitro cell cultures of different explants of hazelnut in 2006 appeared as a new opportunity to recover paclitaxel and other taxanes from C. avellana, with the advantages to use a more abundant and available species than yew.

In addition to the comprehensive review on the chemistry, the biological activities of the hazelnut extracts and of the isolated compounds have been summarized. Furthermore, because the accurate quali-quantitative analysis of phytochemicals is critical to understand the quality parameters of the different parts of C. avellana, analytical works carried out on hazelnuts and byproducts of $C$. avellana have also been reviewed.

\section{Botanical Aspects}

The hazel plant (C. avellana), belonging to the Betulaceae family, is a tree native to Europe and Asia. The natural distribution of C. avellana is restricted to the northern hemisphere, even if it grows in temperate climates like Turkey, Spain, and Italy ( $\triangleright$ Fig. $\mathbf{1}$ ). Although Turkey and Italy remain the major producing countries ( $80 \%$ of the world crop), hazelnut cultivation has spread in recent years to new growing areas including the southern hemisphere [5]. Hazelnut trees, which may grow up to $6 \mathrm{~m}$, exhibit deciduous leaves $6-12 \mathrm{~cm}$ long, softly hairy on both surfaces, and with a double-serrated margin [6]. The kernel, the nut of commerce, is surrounded by a dark brown perisperm and protected by a woody shell [7]. Hazelnuts develop in clusters of 1-12, each separately enclosed in a cup of green leafy cover that encloses about threequarters of the nut [8]. Flowers are produced very early in spring before the leaves and are monoecious, with single-sex catkins; the male catkins are pale yellow and $5-12 \mathrm{~cm}$ long, and the female ones are very small and largely concealed in the buds, with only the bright-red, 1- to 3-mm-long styles visible [9].

The hazelnut cultivars show a high level of genetic diversity for traits such as vigor, growth habits, suckering, nut size and shape, and shell thickness. Cultivars more used in the food industry are Tonda Gentile delle Langhe, also named Tonda Gentile Trilobata, Tonda Gentile Romana, Tonda di Giffoni, S. Giovanni, Mortarella, and Riccia di Talanico, cultivated in Italy; Tombul, Sivri, Palaz, and Fosa, cultivated in Turkey; and Negret and Pauetet produced in Spain. Among Italian cultivars, Tonda di Giffoni and Tonda Gentile delle Langhe gained the European Protected Geographical Indication (PGI) label and are known as "Nocciola di Giffoni" and "Nocciola Piemonte," respectively. U.S. production, especially from Oregon, is principally destined for fresh consumption and only recently has been directed to industrial use [10].

\section{Traditional Medicine Uses}

Persian medicine, a traditional medicine developed on Iran's plateau thousands of years ago, paid special attention to hazelnut providing notable recorded therapeutic experiences in cognitive disorders such as amnesia and dementia based on its own princi- 
ples in etiology and treatment. In some books on Persian medicine, it is specifically emphasized that hazelnut and almond protect the brain tissue and prevent the brain atrophy, improving memory [11].

In Iranian traditional medicine, the leaves of $C$. avellana, known as fandogh, are also largely consumed in the form of infusion as an efficient liver tonic [12]; nowadays, they are also used in folk medicine for the treatment of hemorrhoids, varicose veins, phlebitis, and lower members' edema, as a consequence of their astringency and vasoprotective and anti-edema properties. Galenic preparations of hazelnut leaves have also been used as remedy for ulcers and oropharynx infections. Slight antidysenteric, antifungal, and cicatrizant properties have also been described [13].

In the Swedish traditional medicine, the hazelnut leaves and bark are used for the treatment of pain [14].

\section{Chemistry}

The investigations were mainly focused on differences resulting from cultivars, origin including fresh, raw, and roasted kernels, and on a variety of hazelnut products (hazelnut skin, hard shell, tree leaf, green leafy cover). Herein, in the present work a rationalization of the bioactive specialized metabolites occurring in the hazelnut kernels and other biomasses of $C$. avellana on the basis of the chemical features has been carried out. Phenolics are the most abundant compounds not only in the kernels, but also in other processing byproducts that were reported to contain different derivatives and levels of these compounds. A paragraph focused on the taxane derivatives isolated from C. avellana leaves has also been included.

\section{Phenolic Acids}

The term "phenolic acids" describes phenols that possess 1 carboxylic acid functional group. The naturally occurring phenolic acids can be divided into 2 subcategories: hydroxybenzoic acids and hydroxycinnamic acids. Although the basic skeleton remains the same, the number and positions of the hydroxyl groups on the aromatic ring vary [15].

Several phenolic acids belonging to the hydroxybenzoic acid class were identified in the hazelnut kernel: $p$-hydroxybenzoic acid (1), salycilic acid (2), 4-hydroxysalicylic acid (3), gallic acid (4), vanillic acid (5), and syringic acid (6) [16, 17] ( Fig. 2). Gallic acid (4) represents a main compound of $C$. avellana due to its occurrence in hazelnut kernel, green leafy cover [2], skin, tree leaves [3], and shells [18]. Protocatechuic acid (7) was recently isolated from hazelnut kernel (both fresh and roasted) and hazelnut skin [17] and was identified as the main constituent in the brown skin of hazelnut produced in the United States [2] and also reported in the shells [18]. Vanillic acid (5) was also reported in the hazelnut shells [18], along with methyl gallate (8) and veratric acid (9) [19] ( $\vee$ Fig. 2).

Hydroxycinnamic derivatives as p-coumaric acid (10), caffeic acid (11), ferulic acid (12), and sinapic acid (13) were identified in hazelnut kernel, green leafy cover [2], skin, and tree leaves [3]. m-hydroxycinnamic acid (14), o-coumaric acid (15), and isoferulic acid (16) were identified only in the hazelnut kernel [16,17]. Additionally, compounds 10-13 were described in the hazelnut shells

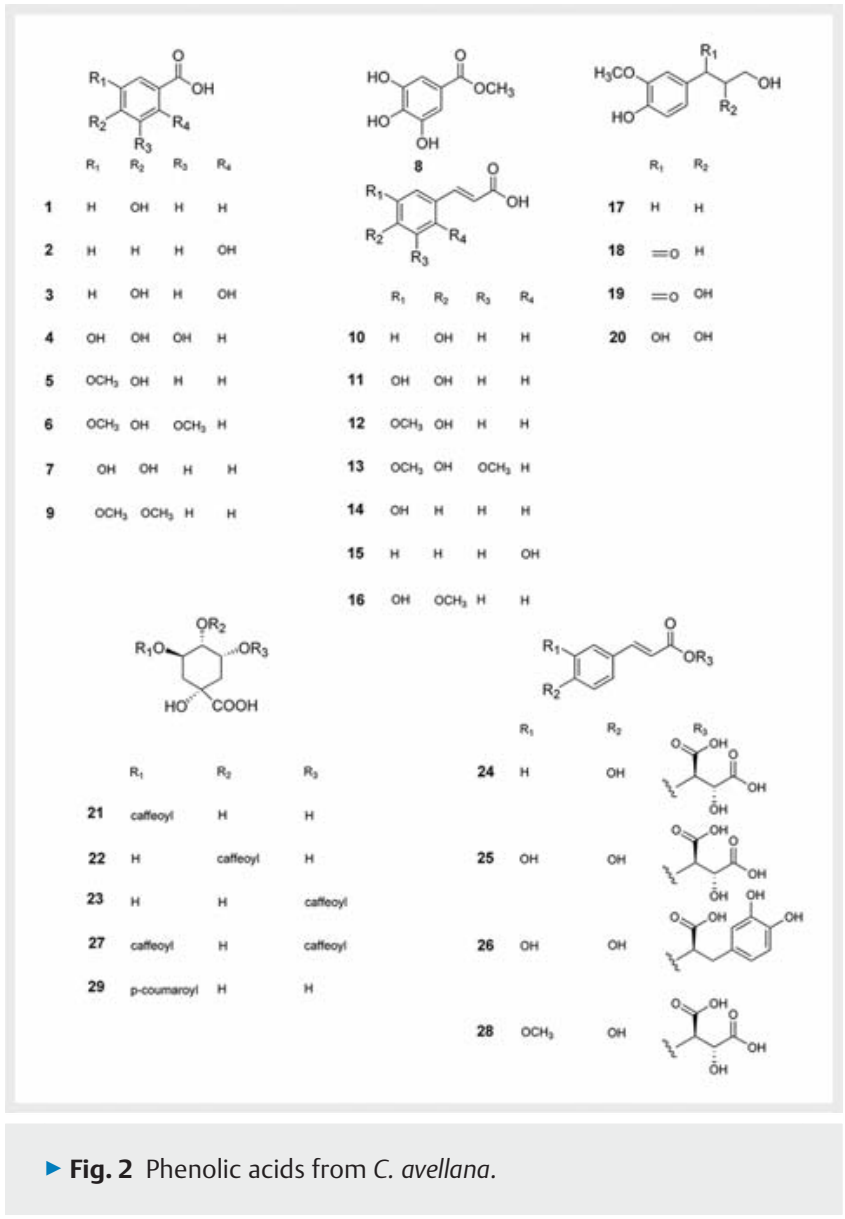

[18] along with dihydroconiferyl alcohol (17) and hydroxypropiovanillone (18) [19], 2,3-dihydroxy-1-(4-hydroxy-3-methoxyphenyl)-propan-1-one (19), and 1-(4-hydroxy-3-methoxy)-1,2,3-propanetriol (20) [20] (॰ Fig. 2).

Phenolic acids may arise in food plants as glycosides or esters with other natural compounds such as sterols, alcohols, glycosides, and hydroxy fatty acids [15]. The hazelnut leaves represented a source of 3-, 4-, and 5-caffeoylquinic acids (21-23), pcoumaroyltartaric acid (24), caffeoyltartaric acid (25) [21], rosmarinic acid (26), and a caffeoyl-hexoside derivative [22], while 3,5-dicaffeoylquinic acid (27) was reported in the leaf cover [8]. As it is evident, these compounds mainly occur in the leaves of hazelnut, although 3-caffeoylquinic acid (21) [23] was also identified in kernel ( $\bullet$ Fig. 2).

Other phenolic acids were tentatively identified by mass spectrometry in the hazelnut shells: galloylquinic acid, coumaroylquinic acid, feruloylquinic acid, along with a pentose ester of coumaric acid and hexose ester of syringic acid [18]. Moreover, compounds such as $p$-coumaroyltartaric acid (24), fertaric acid (28), 3-p-coumaroylquinic acid (29), dihydroxycoumarin, and digalloyl-glucose isomer were tentatively identified in hazelnut samples of Turkish Tombul [17] ( $\triangleright$ Fig. 2).

\section{Flavonoids}

Flavonoids are polyphenolic compounds ubiquitous in nature. They occur as aglycones, glycosides, and methylated derivatives. 
Small amounts of aglycones are frequently present and occasionally represent a proportion of the total flavonoid content in the plant. In > Fig. 3, flavonoids occurring in C. avellana are shown.

The aglycone quercetin (30) was identified in the hazelnut kernels and shells $[17,18]$ and myricetin $(31)$ in the hazelnut kernels, shells, and skins $[17,18,24]$. Flavonoid glycosides reported in hazelnut are all $\mathrm{O}$-glycosides characterized by a sugar portion made up of 1 or 2 units. Kaempferol, quercetin, and myricetin glycosides showed the sugar portion linked to C-3 of the aglycones. Quercetin 3-rhamnoside (32) and myricetin 3-rhamnoside (33) were detected in kernels, shells, leaves, and bark [18,21,22,25] with quercetin 3-rhamnoside (32) also reported in the skins [24], kaempferol 3-rhamnoside (34) in leaves and barks [21,22], quercetin-3-glucoside (35) in kernels and leaves [17,26], and kaempferol 3-glucoside (36) only in kernels [27]. Hyperoside (37) was isolated in the hazelnut leaves [28] and rutin (38) was described in kernels and shells $[18,23]$.

The only investigation carried out on hazelnut flowers evidenced the presence of the previous described compounds 32 , 34 , and 35 , along with quercetin 3-galactosyl-( $1 \rightarrow 2)$-glucoside (39), kaempferol 3-glucosyl-( $1 \rightarrow 2)$-glucoside (40), kaempferol 3-(cis-p-coumaroyl)-rhamnoside (41), and kaempferol 3-(trans-pcoumaroyl)-rhamnoside (42) [9]. Previous phytochemical investigation on C. avellana pollen, strongly connected with the male flowers, reported the presence of quercetin 3-glucosyl-(1 $\rightarrow 2)$ galactoside (43) [29]. More recent investigations highlighted as compound 42 occurred in the hazelnut shells, leaves, and green leafy covers $[8,20,30]$, while compound 41 was described in these byproducts and in the shells [20].

Compounds 33 and 34 were isolated from the hazelnut leafy covers [8]. Moreover, quercetin glucuronide and isorhamnetin-3$\mathrm{O}$-rutinoside (44) were tentatively identified in the hazelnut kernel [17].

Flavanone glycosides were also described in hazelnut: eriodictyol (45) was tentatively identified in the hazelnut kernel [17], taxifolin (46) in the shells [18], and naringin (47) in hazelnut shells and skins [31].

Moreover, the dihydrochalcone glucoside, phloretin 2'-O-glucoside (48), was reported in the hazelnut kernels and shells [18, 25]. Among flavonoids, flavan-3-ols, widely distributed in the plant kingdom, were reported [32]. Catechin (49), epicatechin (50), and epigallocatechin (51) were identified in hazelnut kernels [7], shells [18], and skins [32], and gallocatechin (52) was identified in the skins [24] ( $\triangleright$ Fig. 4). These compounds are present as monomers and as oligomeric and polymeric forms, described in the next paragraph.

\section{Tannins and Proanthocyanidins}

Tannins are a heterogeneous group of high-molecular-weight polyphenolic compounds with the capacity to form reversible and irreversible complexes with proteins, polysaccharides, alkaloids, nucleic acids, and minerals. On the basis of their structural features, it is possible to divide them into 4 major groups: gallotannins and ellagitannins (both known as hydrolyzable tannins), complex tannins, and condensed tannins, also known as proanthocyanidins [15].

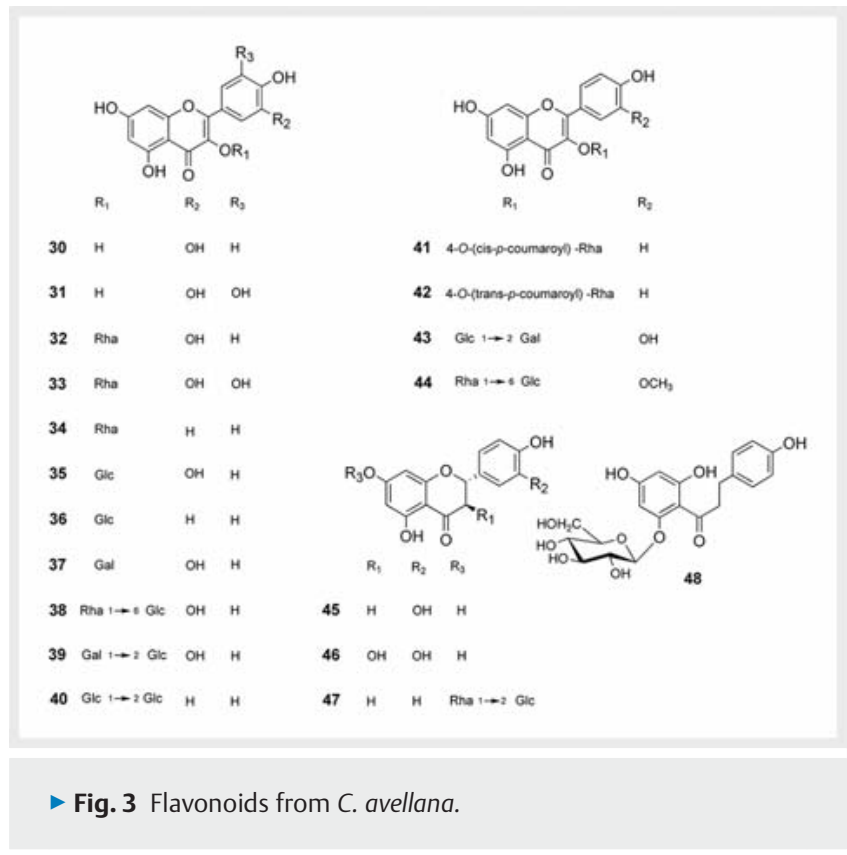

In hazelnut, gallotannins are represented by compounds in which galloyl units are linked to catechin units ( $\bullet$ Fig. 4). Epicatechin gallate (53), epigallocatechin gallate (54), and gallocatechin gallate (55) were described in the hazelnut kernel [33], and epicatechin gallate (53) was identified in the skins [24].

An example of ellagitannin, in which at least 2 galloyl units are C-C coupled to each other, is represented by ellagic acid (56), found in the hazelnut kernel [23] ( Fig. 4).

Five hydrolysable tannins and related compounds ellagic acid hexoside isomer, ellagic acid pentoside isomer, flavogallonic acid dilactone isomer, bis (hexahydroxydiphenoyl)-glucose (HHDPglucose) isomer, and valoneic acid dilactone/sanguisorbic acid dilactone were tentatively identified in hazelnut samples by Pelvan [17].

Condensed tannins or proanthocyanidins, widespread in hazelnut and its byproducts, are oligomers or polymers classified in procyanidins, propelargonidins, or prodelphinidins on the basis of the flavan-3-ol unit (epi)catechin, (epi)afzelechin, or (epi)gallocatechin, respectively [25]. In detail, in relation to the interflavanic bond nature, B-type procyanidins are those in which monomers are linked through the C-4 position of the top unit and the C- 6 or $\mathrm{C}-8$ positions of the terminal unit, the $\mathrm{C}-4$ to $\mathrm{C}-8$ isomers being more abundant than the C-4 to C-6 ones. On the other hand, Atype procyanidins contain an additional ether type bond between the $\mathrm{C}-2$ position of the top unit and the hydroxyl group at C-5 or C-7 of the lower unit [32].

Procyanidins A2 (57), B1 (58), and B2 (59) [7,33] along with other procyanidin dimers and trimers were identified in hazelnut kernels $[10,25]$ ( $\bullet$ Fig. 4). Four isomers of B-type procyanidin were tentatively identified in hazelnut shells [18]. Investigation of hazelnut skins reported the occurrence of oligomeric proanthocyanidins with a degree of polymerization up to 10 , mainly constituted by B-type oligomers of (epi)catechin. Also, (epi)gallocatechin and gallate derivatives were identified as monomeric units, 


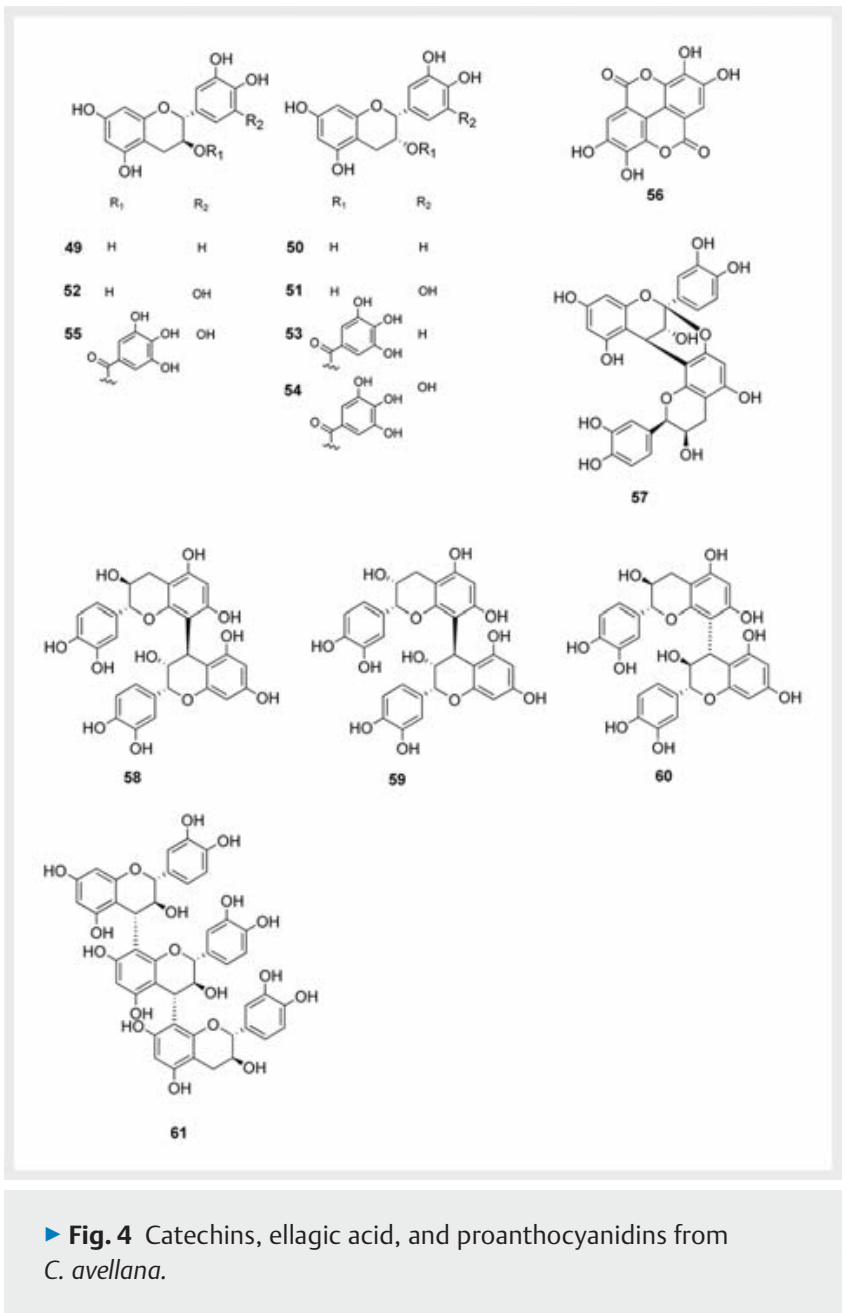

and A-type proanthocyanidins were detected as minor constituents [34]. Del Rio reported in hazelnut skins 9 B-type dimers of procyanidins, among which were procyanidins B1 (58), B2 (59), B3 $(60), 3$ procyanidin gallate dimers, and 5 procyanidin trimers ( $\bullet$ Fig. 4). Moreover, 1 trimer was tentatively identified as procyanidin C2 (61) [24] (॰ Fig. 4).

\section{Diarylheptanoids}

Diarylheptanoids, a class of natural products based on the 1,7-diphenylheptane skeleton, occur frequently in plants belonging to the Betulaceae family. They can be divided into linear or cyclic compounds. There is a smaller number of cyclic diarylheptanoids that are formed from the corresponding linear type by phenolic oxidative coupling, either C-C coupling leading to meta,metabridged biaryls or $\mathrm{C}-\mathrm{O}$ coupling leading to bridged diaryl ethers. Thus, they can be classified into 3 subgroups: linear diarylheptanoids, cyclic diarylheptanoids, and cyclic diaryletherheptanoids ( $\bullet$ Fig. 5). Hazelnut leaves represent a source of diarylheptanoids. The first report of linear diarylheptanoids focused on the identification of 11,7-bis-(3,4-dihydroxyphenyl)-4-hepten-3-one (hirsutenone) (62), 1,7-bis-(4-hydroxyphenyl)-4,6-heptadien-3-one (63), 5-hydroxy-1-(4-hydroxyphenyl)-7-(3,4-dihydroxyphenyl)heptan-3-one (64), 5-hydroxy-1-(3,4-dihydroxyphenyl)-7-(4-hy- droxyphenyl)-heptan-3-one (65), 7-bis-(3,4-dihydroxyphenyl)-4hepten-3-one-hexoside, 1,7-bis-(4-hydroxyphenyl)-4,6-heptadien-3-one-hexoside, and 1,7-bis-(4-hydroxyphenyl)-4-hepten-3one-hexoside [22], the latest tentatively identified by MS. A further linear diarylheptanoid, (3R,5R)-3,5-dihydroxy-1,7-bis(4-hydroxy- phenyl) heptane 3-O- $\beta$-D-glucopyranoside (66), was isolated in the hazelnut leaves [35].

More recently, cyclic diarylheptanoids were isolated from hazelnut leaves, leaf covers, flowers, and shells of the Italian cultivar Tonda di Giffoni. For these reasons, the compounds were named giffonins. The cyclic diaryletherheptanoids named giffonins $\mathrm{A}-\mathrm{H}$ (67-74), and J-K $(75,76)$ were isolated from the hazelnut leaves while giffonins Q-S (77-79) were isolated from the hazelnut flowers $[6,9,30]$.

The cyclic diarylheptanoids named giffonins I (80), L-P (81$85), T(86)$, and $U(87)$ along with carpinontriol $B(88)$ were isolated from the hazelnut leaves; giffonins I (80), T (86), U (87), and carpinontriol B (88) were also isolated from hazelnut leaf covers; giffonin P (85), carpinontriol B (88), and giffonin $V(89)$ were also found in the hazelnut shells $[6,8,20,30]$. Moreover, giffonin I (80) along with alnusone (90) were described in the hazelnut flowers [9]. Chemically, giffonins A-I $(67-74,80)$ are characterized by the presence of only 1 stereogenic center on the heptyl moiety; for these compounds the absolute configuration was established through the application of the modified Mosher's method [6]. For giffonins J-P $(75,76,81-85)$, possessing at least 2 stereogenic centers on the heptyl unit, a combined (quantum mechanical)/NMR approach based on the comparison of the experimental ${ }^{13} \mathrm{C} /{ }^{1} \mathrm{H}$ NMR chemical shift data and the related predicted values was used to establish the relative configurations [30], and for giffonins $T$ (86) and $U$ (87), this approach was followed by the comparison of the experimental electronic circular dichroism (ECD) curves with the TDDFT calculated curves to establish the absolute configurations [8].

\section{Lignans}

Lignans are a class of secondary metabolites derived from 2 phenylpropanoid units linked through a C-C bond between $\mathrm{C} 8$ and C8' of the side chain carbon atoms. A careful literature search highlights as lignans and related compounds, widely distributed in the plant kingdom, can show a remarkable structural diversity, although they arise from the relatively simple basic skeleton of 2 phenylpropanoids [36]. Neolignans are characterized by 2 phenilpropanoid units linked by a bond other than a C8-C8' bond. These compounds were described in the hazelnut shells as erythro(7S,8R)-guaiacylglycerol- $\beta$-O-4'-dihydroconiferyl alcohol (91), erythro-(7S,8R)-guaiacylglycerol- $\beta$-coniferyl aldehyde ether (92), erythro-(7R,8S)-guaiacylglycerol- $\beta$-O-4'-dihydroconiferyl alcohol (93), threo-1,2-bis(4-hydroxy-3-methoxyphenyl)-1,3-propandiol (94), ceplignan (95), ficusal (96), ent-cedrusin (97), dihydrodehydrodiconiferyl alcohol (98), and balanophonin (99) [20] (• Fig. 6).

\section{Taxanes}

An article published in 2006 reported the recovery of taxanes in in vitro cell cultures of different explants of hazelnut [37] ( $\bullet$ Fig. 7). The explants were used to optimize the protocol for inducing in vitro callus, an undifferentiated tissue from which suspension cell 


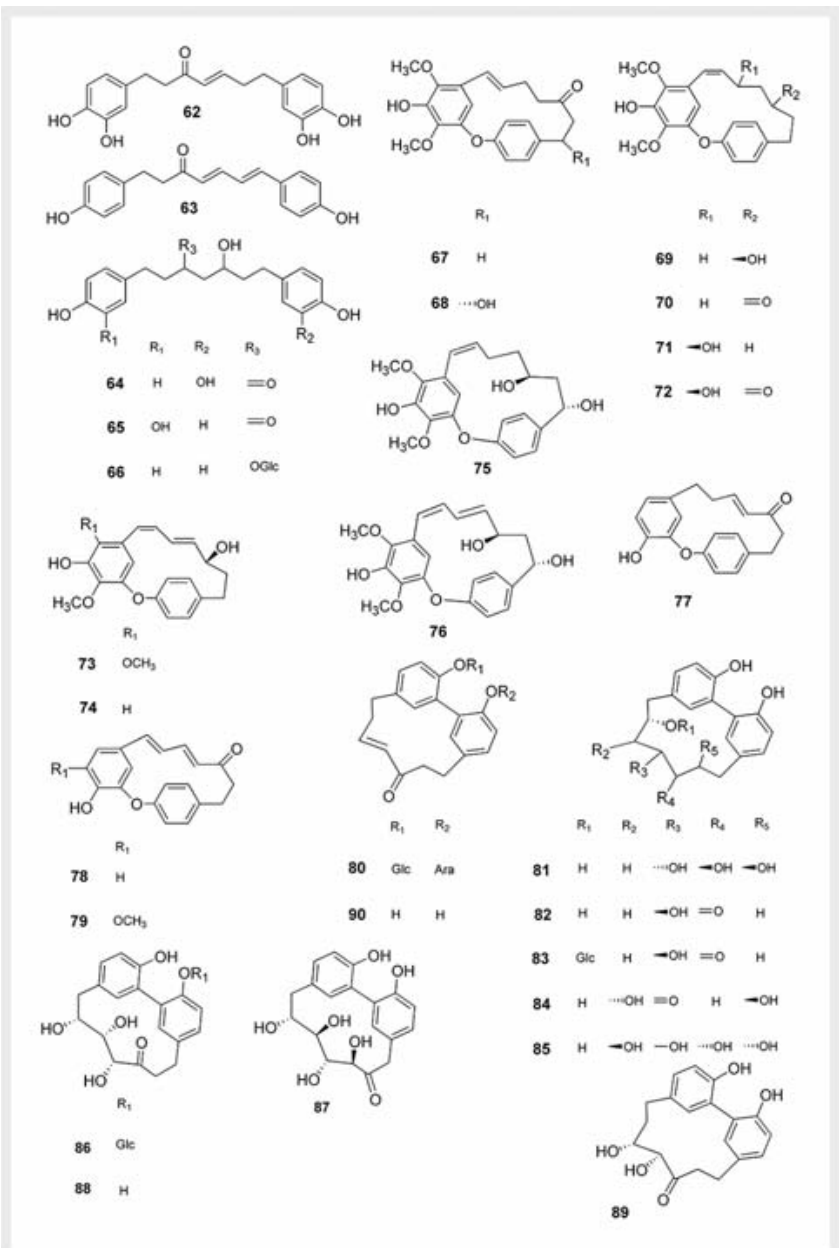

- Fig. 5 Diarylheptanoids from C. avellana.

cultures were obtained. The media recovered from suspension cell cultures contained taxanes as paclitaxel (100), 10-deacetylpaclitaxel (101), and 10-deacetylbaccatin III (102). This paper suggested that hazel species possess the metabolic pathway(s) for taxane biosynthesis [37]. Later, hazelnut leaves and shells were investigated for the presence of taxanes [38]. The extracts were found to contain paclitaxel (100), 10-deacetylpaclitaxel (101), 10-deacetylbaccatin III (102), baccatin III (103), 7-xylosylpaclitaxel (104), 10-deacetyl-7-xylosylpaclitaxel (105), 7-epipaclitaxel (106), 10-deacetyl-7-epipaclitaxel (107), 10-deacetyl-7-xylosylcephalomannine (108), cephalomannine (109), paclitaxel C (110), 10-deacetyl-7-xylosylpaclitaxel C (111), and taxinine M (112) ( $\vee$ Fig. 7). The isolated taxanes were quantified with the exception of compounds 101, 104, 105, 107-109, 111, and 112, which were not individually quantified due to the difficulties in separation under the chromatographic condition used [38]. This investigation highlighted as the level of total taxanes in leaves was higher than in shells collected in the same period from the same plants [38].

Small amounts of paclitaxel (100), 10-deacetyl baccatin III (102), baccatin III (103), and cephalomannine (109) were also isolated from Tombul hazelnut hard shells, green leafy covers, and
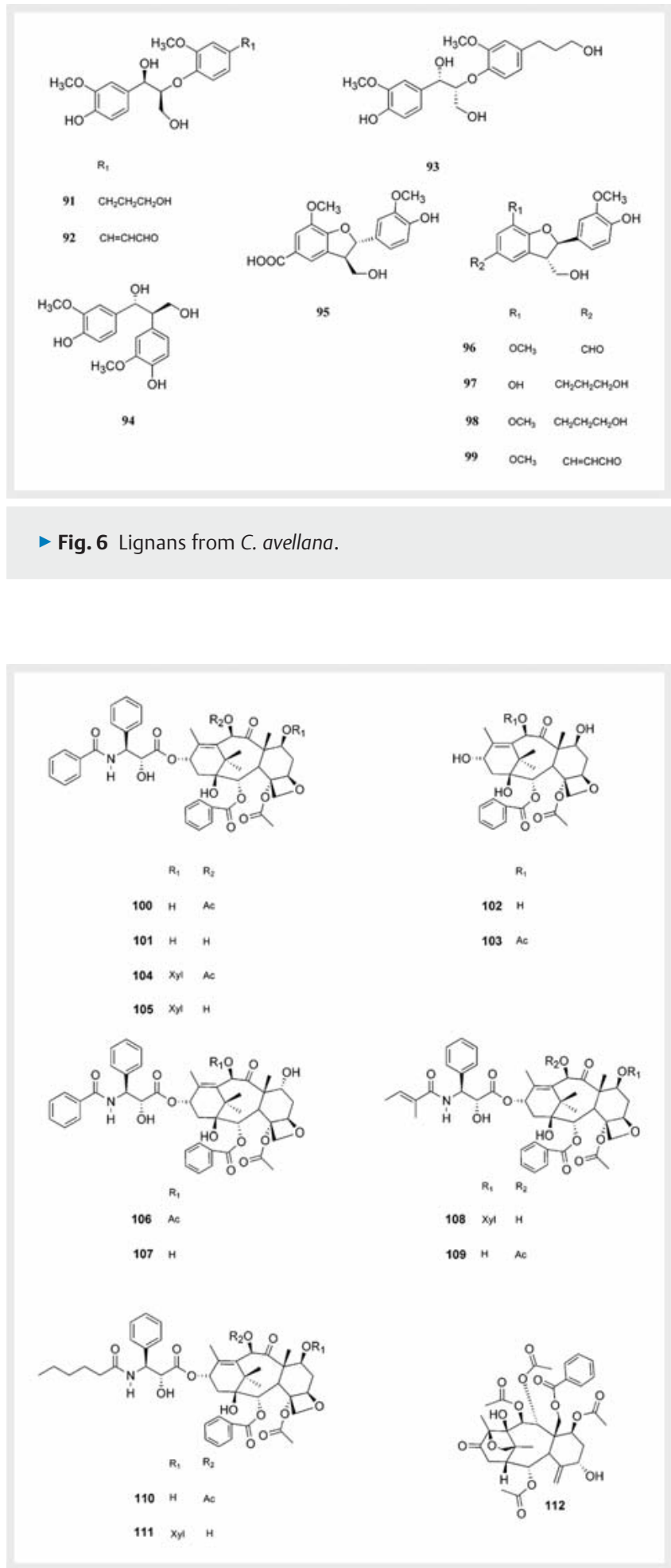

- Fig. 7 Taxanes from C. avellana.

leaves [39]. On the basis of the recovery of taxanes in the hazelnut leaves and shells, investigations were also performed on the kernels. None of the previous described taxanes were found in the hazelnut kernels $[39,40]$, except 1 compound tentatively identified as 7-epi-paclitaxel [40]. 


\section{Hazelnut Volatiles}

Hazelnuts are famous for their distinctive taste and aroma. Compounds including ketones, aldehydes, pyrazines, alcohols, aromatic hydrocarbons, furans, pyrroles, terpenes, and acids were detected in fresh and roasted hazelnuts [41-43]. Among the several volatiles compounds, 5-methyl-(E)-2-hepten-4-one (filbertone) was reported as primary odorant (nutty-roasty and hazelnut-like) of roasted hazelnuts. The different roasting conditions were reported to influence the aroma chemical composition: in the roasted hazelnuts were identified more volatile compounds than in the fresh hazelnuts [43].

\section{Biological Activity}

\section{Antioxidant Activity}

Phenolic compounds have been reported to reduce the risk of cancer, heart disease, and several neurodegenerative diseases; to prevent and delay many age related pathologies; to inhibit plasma platelet aggregation, cyclooxygenase activity, and histamine release; and to exert antibacterial activity $[44,45]$. The benefits toward many of these conditions arise in part from their antioxidant properties being able to act as direct and indirect antioxidants via mechanisms mainly involving free radical scavenging or neutralizing other oxidants $[46,47]$.

Purified phenolic compounds, phenolic-enriched fractions, along with the whole polar extracts occurring in both hazelnut kernel and its byproducts have been reported to exert strong antioxidant activities $[1,4,48]$. General lines regarding the antioxidant activity exerted by the phenolics-rich extracts obtained from kernels and byproducts of $C$. avellana will be drawn, taking into account that the hazelnut extracts were obtained by different extraction methods and were submitted to different antioxidant assays.

The total antioxidant activities of the extracts obtained from hazelnut kernels, natural and roasted, with or without skins, differing for geographical origins, varieties, and storage conditions, have been measured $[7,17,27,49-58]$. Mostly, no statistical differences in total antioxidant activity have been observed among samples of unroasted and roasted kernels without skins; the thermal processing had a light negative trend in antioxidant efficacy mainly due to the absence of skins $[1,27,50,52,59]$. The effects of kernel roasting by 2 different processing methods, microwave and microwave-assisted hot air, revealed in both cases an increase of antioxidant activity at the rising of roasting time and temperature was observed, maybe explainable according to the formation of new Maillard reaction products having antioxidant activity [56, 60].

When the skins were retained on the kernels, a general trend showing a higher total antioxidant ability for the extracts could be observed, suggesting a direct contribution of skin phenolic content, above all consisting of proanthocyanidins, although there is no unanimous opinion about this relationship and this hypothesis has been frequently discussed and opposed $[3,4,17,24$, $25,32,34,52,55,58,59,61,62]$.

Mostly, apart the extracts obtained from skins, also the extracts of the other C. avellana byproducts (shells, green leafy cov- ers, flowers, and leaves) exhibited stronger activities than hazelnut kernel $[2,3,6,8,9,19-21,63]$. However, the antioxidant ability of the phenolic compounds isolated from the extracts of these byproducts was in some cases higher than that of the extracts themselves. For example, the diaryletherheptanoids giffonin $\mathrm{D}$ (70) and $\mathrm{H}(74)$ at $10 \mu \mathrm{M}$ reduced both $\mathrm{H}_{2} \mathrm{O}_{2}{ }^{-}$and $\mathrm{H}_{2} \mathrm{O}_{2} / \mathrm{Fe}^{2+}$-induced lipid peroxidation in TBARS assay by more than $60 \%$ and $50 \%$, respectively, being more active than methanol extract $(38 \%$ at $10 \mu \mathrm{g} / \mathrm{mL}$ in both assays) and curcumin, used as reference compound [30]. Analogously, in the same in vitro test, giffonin $R$ (78) and $S$ (79) at $10 \mu \mathrm{M}$ exhibited an inhibition of lipid peroxidation by more than $50 \%$ and about $35 \%$, respectively, resulting more active than both $C$. avellana flower extract, from which they were isolated, and curcumin [9]. When tested for their antioxidant ability by TBARS assays, all compounds isolated from methanol extracts of leaves, flowers, and green leafy covers of Italian Tonda di Giffoni hazelnut exhibited an inhibition of lipid peroxidation at least comparable to curcumin $[6,8,9]$. The antioxidant activity evaluated by TEAC assay for diarylheptanoids, neolignans, phenylpropanoids, and flavonoids isolated from the hazelnut shells of the same C. avellana (cultivar Tonda di Giffoni) highlighted higher free radical scavenging activity (similar to that shown by quercetin 3-O-glucoside used as reference compound) for kaempferol 3(4"'-cis-p-coumaroyl)-rhamnoside (41) and kaempferol 3-(4"'trans-p-coumaroyl)-rhamnoside (42), suggesting that the coumaroyl moiety improves the radical-scavenging capacity, while diarylheptanoids giffonin $\mathrm{P}(85)$, carpinontriol $\mathrm{B}(88)$, and giffonin $V(89)$, possessing similar chemical features, characterized by the presence of 2 phenolic groups, showed a weak radical-scavenging capacity as well as threo-1,2-bis(4-hydroxy-3-methoxyphenyl)1,3-propandiol (94) [20]. The antioxidant activities by TEAC assay have been evaluated also for both $n$-butanol extracts of raw and roasted kernels (without skins), obtained from the same plant cultivar, and phenolics compounds occurring in them [27]. Among the tested compounds, ellagic acid displayed the highest activity, the flavonoid derivatives myricetin 3-rhamnoside (33) and quercetin 3-glucoside (35) showed a free radical scavenging activity comparable to that displayed by the reference compound quercetin, while both kernel extracts and diarylheptanoids giffonin M (82), and Q (77) showed the weakest TEAC values.

The additive and synergistic effect of phenolic compounds (i.e., neolignans, phenolic acid derivatives, and cyclic diarylheptanoids) isolated from the polar extract of the hazelnut shells obtained as waste product of industrial processing of 2 other Italian varieties (Mortarella and Lunga San Giovanni) was instead invoked to explain the higher free radical scavenging activity shown in DPPH assay by the extract when compared with the metabolites therefrom isolated [19].

\section{Antiproliferative Effects}

Considering the increasing evidence suggesting that inflammation plays a pivotal role in a multitude of chronic diseases, including cancer, and that there exists a close correlation between antioxidant ability and anticancer effects [64], the antiproliferative effects of both extracts and phenolic compounds derived from hazelnut kernels and byproducts have been largely studied. Moreover, the presence of small quantities of Taxol and taxanes in 
C. avellana $[37,38]$ and above all the consideration that C. avellana cell culture extracts were more effective than pure Taxol against human cancer cell lines, have increased the investigations on both the extracts and its secondary metabolites, mainly phenolics.

Thus, the polar extracts of shells, leaves, and green leafy covers of the Italian C. avellana cultivar Tonda di Giffoni, affording numerous metabolites belonging to diarylheptanoids class along with flavonoids, neolignans, and phenylpropanoids, have been investigated for their cytotoxic activities against different human cancer cell lines (human lung adenocarcinoma, A549; human epithelioid cervix carcinoma, Hela; human skin fibroblasts, HaCat; human B lymphoma, DeFew; human osteosarcomas, U2Os and SAOs) [8, $20,30]$. Neither single phenolics (in a range of concentrations between 10 and $100 \mu \mathrm{M}$ ) nor the methanol extracts (at $500 \mu \mathrm{g} / \mathrm{mL}$ and $250 \mu \mathrm{g} / \mathrm{mL}$ ) of these hazelnut byproducts caused a significant reduction of the number of cancer cell lines. The study of Esposito et al. reported in in vitro MTT assay a low inhibitory effect on the growth of human cancer cell lines of primary and metastatic melanoma (A375 and SK-Mel-28, respectively) and cervix carcinoma (HeLa) for the methanol extract, the neolignans balanophonin (99) and ent-cedrusin (97) and the phenol derivative gallic acid (4) obtained from hazelnut shells of 2 others Italian varieties (Mortarella and Lunga San Giovanni) [19]. The expression of cleaved forms of caspase-3 and poly(ADP-ribose) polymerase-1 (PARP-1) suggested that the shell extract induced apoptosis through caspase-3 activation in both SK-Mel-28 and HeLa cell lines. In 2008, Ottaggio et al. analyzed the methanol extracts from leaves and shells of $C$. avellana, reporting the presence in both of taxanes such as paclitaxel (100), 10-deacetylbaccatin III (102), baccatin III (103), paclitaxel C (110), and 7-epipaclitaxel (106), with leaves containing a higher level of taxanes than shells collected in the same period from the same plants [38]. In the study, the biological activities of shell hazel extracts were investigated by evaluating their ability to inhibit metaphase to anaphase transition in human cancer SK-Mes-1 (squamous cell lung carcinoma) cell culture. A decrease in the anaphase/metaphase ratio was observed in both Taxol and shell extract ( 0.026 vs. 0.054 , respectively) treated cells.

Both leaf and stem extracts of 3 different Spanish hazel trees significantly reduced viability of the 3 human derived cancer cell lines (HeLa; liver hepatocellular cells, HepG2; human breast adenocarcinoma cell, MCF-7) [35]. Among the 2 tested extraction methods, the maceration with methanol reduced cell viability to a greater extent than taxane extraction methods and in particular methanol leaf extracts promoted a higher reduction in viability of all cell lines assayed than stem extracts. Fractionation of methanol leaf extracts led to the purification and identification of 2 compounds, (3R,5R)-3,5-dihydroxy-1,7-bis(4-hydroxy- phenyl) heptane 3-O- $\beta$-D-glucopyranoside (66) and quercetin 3-rhamnoside (32), that decreased viability of HeLa and HepG2 cells to a greater extent than MCF-7 cells.

Moreover, Li and Parry (2011) examined the extracts obtained from both roasted and raw Turkish and Oregon hazelnuts as shelled nuts (with or without skins) and skins (raw and roasted) by testing in vitro the antiproliferative effects against human colon cancer HT-29 cell line [58]. At $6 \mathrm{mg} / \mathrm{mL}$ media Oregon roasted hazelnut skin extract significantly inhibited the growth of HT-29 cells by $96 \%$ following $4 \mathrm{~d}$ of treatment, and a similar result was seen from the Turkish roasted skin hazelnut extract with an inhibition of growth of the HT-29 cells by $89 \%$.

\section{Antimicrobial, Antifungal, and Anthelmintic effects}

Antimicrobial natural compounds are receiving a growing interest because of consumer pressure on the food industry to avoid chemical preservatives and the increasing resistance to antibiotics. Several investigations on hazelnut kernels and byproducts have been carried out with the aim to evaluate the antimicrobial activity of extracts and single compounds on the basis of the antimicrobial capacity described for phenolic compounds $[65,66]$.

Thus, in their work Cerulli et al. evaluated the antimicrobial activity of both methanol extract of leafy covers of $C$. avellana Tonda di Giffoni and cyclic diarylheptanoids, quinic acid, flavonoid, and citric acid derivatives therefrom isolated against the Gram-positive strains Bacillus cereus and Staphylococcus aureus and the Gram-negative strains Escherichia coli and Pseudomonas aeruginosa [8]. The antimicrobial assays established that the cyclic diarylheptanoids giffonin $U(87)$ and carpinontriol $B(88)$ were the most effective against the tested strains, causing at $40 \mathrm{\mu g} /$ disk the formation of zones of inhibition completely comparable to those obtained with tetracycline at $7 \mu \mathrm{g} /$ disk used as positive control.

The antimicrobial capacity analysis against Gram-positive (B. cereus, B. subtilis, S. aureus) and Gram-negative bacteria (P. aeruginosa, E. coli, Klebsiella pneumoniae), and fungi (Candida albicans, Cryptococcus neoformans) of aqueous extracts of kernels of 3 different C. avellana cultivars produced in Portugal (cv. Daviana, Fertille de Coutard, and M. Bollwiller) [57] revealed high antimicrobial activity only against Gram-positive bacteria, Gram-negative bacteria and fungi being resistant to the tested extracts at all the assayed concentrations. B. cereus was more susceptible (MICs of $0.1 \mathrm{mg} / \mathrm{mL}$ for cv. Daviana and M. Bollwiller) than the other Gram-positive bacteria. These extracts had a similar antimicrobial activity to leaf extracts of the same cultivars [21], which were effective at low concentrations $(\sim 0.1 \mathrm{mg} / \mathrm{mL})$. A good level of antibacterial activity (MIC $125 \mu \mathrm{g} / \mathrm{ml}$ ) against the Listeria monocytogenes strain was also demonstrated for tannins enriched fractions obtained from the acetone extract of $C$. avellana kernels from Poland [67].

By microbroth dilution method the antifungal effect against C. albicans SC5314 of both the extract prepared from roasted hazelnut skins by maceration and the proanthocyanidins-enriched fraction therefrom obtained was tested, reporting the first an antimycotic activity with $\mathrm{MIC}_{2}$ of $3.00 \mu \mathrm{g} / \mathrm{mL}$ and $\mathrm{MIC}_{0}$ of $5.00 \mu \mathrm{g} / \mathrm{mL}$ at $48 \mathrm{~h}$, and the second a higher activity $\left(\mathrm{MIC}_{2}\right.$ $0.10 \mu \mathrm{g} / \mathrm{mL}$ and $\mathrm{MIC}_{0} 0.50 \mu \mathrm{g} / \mathrm{mL}$ ), suggesting that the antifungal activity can be correlated to PAs content [34]. Tannins-enriched fractions obtained from extracts of hazelnut skins showed anthelmintic effects against the nematode parasite Ascaris suum by reducing the migratory ability of newly hatched third-stage larvae and the motility and survival of fourth stage larvae recovered from pigs [68]. Furthermore, the authors provided evidence that the strength of the anthelmintic effect is both related to the polymer size of the tannin molecule and to the identity of the monomeric structural units of tannin polymers. 


\section{Prebiotics vs. Antimicrobial Effects}

Prompted by the finding that the intake of flavonol-rich foods has been shown to modify the composition of the gut microbiota, exerting prebiotic-like effects [69], Montella et al. in 2013 investigated the possibility to prepare novel bioactive extracts with potential prebiotic activity from hazelnut skins in order to promote the growth of 2 well-known probiotic bacteria, Lactobacillus plantarum P17630 and Lactobacillus crispatus P17631 [62]. Thereby samples of hazelnut skins obtained from Tonda Gentile Trilobata (Piedmont) were extracted yielding a total phenolic extract along with a solid residue, from which both a soluble and an insoluble dietary fiber fraction were obtained. The total phenolic extract was in turn fractionated on C-18 SPE cartridges yielding 3 fractions enriched in phenolic acids, monomeric/oligomeric flavan-3ols, and polymeric proanthocyanidins. The antimicrobial effect against the 2 Lactobacillus strains was then assayed for the 4 different polyphenols-rich extracts and the 2 fiber fractions. Both soluble and insoluble dietary fiber obtained from hazelnut skins showed a prebiotic activity towards L. crispatus P17631 and L. plantarum P17630, allowing to improve the growth during in vitro fermentation. On the contrary, free phenolic substances (especially belonging to oligomeric and polymeric proanthocyanidin classes) proved to negatively influence the positive fiber effect, in particular showing, at all tested concentrations, a strain-specific antimicrobial effect against $L$. crispatus P17631, with no significant influence on L. plantarum P17630 bacterial growth.

\section{Protective Effect Against Neurochemical Alterations in Alzheimer's-Type Neurodegeneration}

With the aim to examine the potential nutraceutical benefits of hazelnut in Alzheimer's neurodegenerative disease, the in vitro enzyme inhibition properties of hazelnut samples grown in 2 regions, Piedmont in Italy and the Black Sea region in Turkey, along with the possible neuroprotective effects of both hazelnut varieties on catecholamine levels in rat cerebral cortical specimens challenged with amyloid $\beta$-peptide by ex vivo experiments were evaluated [53]. In particular, in the in vitro enzyme inhibition assays, carried out against cholinesterase, tyrosinase, amylase and glucosidase and performed in agreement with the enzyme inhibitory theory considered as one of the most useful therapeutic ways for managing global health problems including Alzheimer's disease, Turkish hazelnut methanol extracts had a more considerable enzyme inhibitory potential than Italian ones. Ex vivo experiments proved that both hazelnut varieties were able to mitigate neurochemical alterations in Alzheimer's neurodegeneration in rodents by increasing norepinephrine level and reducing dopamine level consistently. The reduction in tissue levels of dopamine was related to increased release as well as to the stimulatory effects on dopamine release induced by ascorbic acid, which is significantly present in hazelnuts and that could enhance the synthesis of norepinephrine from dopamine. The authors also hypothesize that increased levels of norepinephrine can be linked to the high hazelnut polyphenolic and flavonoid content that could be responsible of the blunting effect on norepinephrine levels induced by amyloid $\beta$-peptide treatment, ex vivo, and also that could induce norepinephrine signaling stimulation, mitigating effects against age-related cognitive and motor decline $[53,70]$.

\section{Anticonvulsant Properties}

The synergistic effect of biologically active compounds occurring in leaves of $C$. avellana collected in Ukraine has been invoked to explain the high anticonvulsant properties shown by the dry aqueous extract [71]. In fact, in mice subjected to pentylenetetrazoleinduced seizures the aqueous extract of hazelnut leaves, as well as the reference drug sodium valproate, showed the most pronounced anticonvulsant activity respect to the other hydroalcoholic extracts tested, resulting in a significant increase in the latency period of the first seizure occurrence, reducing lethality and duration of the convulsive period in the group. Both dry ethanol (50\%) and ethanol (96\%) extracts did not practically differ from each other in their anticonvulsant activity, rather showing a decrease of anticonvulsant effect with the increase of ethanol concentration.

\section{Protein Precipitating Capacity}

By forming complexes with proteins, such as salivary proline-rich proteins, polyphenols are largely responsible for astringency [72].

Several studies reported that polyphenol-protein interactions determine a masking effect on the free radical scavenging activity of polyphenols [72,73], thereby rendering of great importance to investigate the affinity of polyphenols for proteins.

With this aim, Pelitli et al. analyzed raw Turkish Tombul hazelnuts with skins. Extracts of defatted hazelnut were fractionated into low-molecular weight and high-molecular weight phenolics, and low-molecular weight fraction was further purified to remove sugars and organic acids [74]. The crude extract and its fractions were then tested by measuring their protein precipitating capacity using 2 different proteins, BSA and gelatin, which were effectively precipitated by high-molecular weight fraction. The optimum $\mathrm{pH}$ for precipitation of polyphenols with BSA and gelatin ( $\mathrm{pH} 4.0$ and $\mathrm{pH} 5.0$, respectively) were similar to the values already reported. Hazelnut polyphenols displayed a greater affinity for gelatin, having a conformational open structure, than for BSA, characterized by a compact globular structure. The differences in the affinities could be due to the differences in the ability of binding sites for polyphenol adsorption as well as the net charge of the proteins used in the study.

\section{Antiglycative Effect}

The inhibitory effect of the aqueous extract of Turkish hazelnut kernel (Ankara) against AGEs formation has been also evaluated [75]. AGEs are the final products derived from the nonenzymatic glycation process. Because they are involved in the development of several health complications associated with diabetes and ageing, extracts with anti-AGE ability could mitigate the effects of age-related pathologies. By measuring the fluorescence intensity and using aminoguanidine as an AGE inhibitor, Mesías et al. ascertained the extract inhibitory effects, at $25 \mathrm{mg} / \mathrm{mL}$, on AGE formation in BSA-glucose and BSA-methylglyoxal (MGO) assays. The extract exhibited a more than $40 \%$ anti-AGE activity in protein-glucose assay when compared with that of amino guanidine (93\%). In BSA-MGO assay hazelnut extract had no inhibitory activity. No relationship between antioxidant and phenolic compound content and antiglycative activity of the extracts was found. This finding led the authors to conclude that other hydrophilic constituents in 
addition to phenolic acids must be involved in the antiglycative activity of the aqueous hazelnut kernel extract.

\section{Analytical Analysis}

Accurate quantitative analyses of phytochemicals, mainly of phenolics, are critical to understand the quality parameters of the different parts of $C$. avellana.

The content of phytochemicals in hazelnuts and other parts of C. avellana generally varies considerably by cultivar, genotype, and pre- and post-harvesting factors. Genotype strongly influences the content of phenolic acids, flavonoids, stilbenes, and phytosterols [25]. Jakopic described that the total phenolic content oscillates from 70 to $478 \mathrm{mg} / \mathrm{kg}$ kernels, with an average of $189.5 \mathrm{mg} /$ $\mathrm{kg}$ among several different hazelnut genotypes. Environmental factors that can be grouped in climatic factors (soil, sun exposure, precipitation) and agronomic factors (organic or conventional cultivation, irrigation, fertilization) can regulate the biosynthesis of secondary metabolites and among them of phenolic compounds. In addition, the environmental stress strongly influences polyphenol biosynthesis because of the ecological function of phenolic compounds. The only information occurring in literature is relative to the total phenolic content and its variability connected with environment, so no advanced phytochemical analysis was reported [76]. Also, effects of agronomic practices (organic versus conventional, for example) on hazelnut metabolomics have not been explored to date. After harvesting, nuts are subjected to different treatments, including sterilization, whitening, roasting, radiation, and packaging. Technological approaches also show quali-quantitative effects on the phytochemicals content. Schmitzer et al. [59] reported that roasting had a negative effect on individual phenolics but not on the total phenolic content and total antioxidant capacity of hazelnuts. They found that roasting decreased the amount of protocatechuic acid (7), phloretin-2-O-glucoside (48), catechin (49), and epicatechin (50) in hazelnuts. The medium content of each single metabolite is reported in $>$ Table 1, based on literature and the database "Phenol-Explorer" [77], which can represent a guide to the single phenol content in different parts of $C$. avellana.

Methods focused on hazelnut quality and typization are mostly based on phenotypic observations and genetic fingerprint. In recent years, genetic approaches are complemented with innovative analytical methodologies that could suitably integrate wellestablished protocols for authentication [10]. Excluding approaches used for hazelnut typization based on the analysis of volatile constituents by GC-MS or other modern approaches based on NMR in combination with multivariate data analysis [78, 79], most of the methods used for metabolomics of hazelnut are principally based on LC and LC-MS methods.

\section{Extraction}

Extraction of phytochemicals is necessary before their quali-quantitative analysis. The choice of solvent significantly determines their amount [48]. Organic solvents, classically methanol, acetone, acetonitrile, or ethyl acetate, have been employed to extract polyphenols from the different parts of $C$. avellana. The different solvent may explain some of the inconsistency in the amount of phytochemicals from the same plant matrix.

As pre-treatment, due to the high percentage of lipids, hazelnuts are commonly milled and washed with a nonpolar solvent (i.e., hexane) to remove fats. This treatment has been carried out by solid-liquid extraction [10] or by using a Soxhlet method [61]. After defatting, in most of the papers the extraction technique exploited for phenolic compounds was the conventional solid-liquid extraction, generally supported by an ultrasound bath improving the mass transfer. Ghirardello et al. explored different solvent mixtures ( $80 \% \mathrm{v} / \mathrm{v}$ ethanol, methanol, and acetone), at different temperature, with the result that a mixture of $80 \% \mathrm{v} / \mathrm{v}$ acetone/water was the most effective in extracting both benzoic and cinnamic acid derivatives [33]. Extraction of phenolics from kernels was also explored in 2018 by Fanali et al. Phenolic compounds in their study were recovered applying 2 different extraction approaches, namely UA-SLE and SPE. Different solvents were assessed, determining total phenolic content and flavonoids content in addition to antioxidant activity. The best extraction conditions, giving the highest value of total phenolic content in addition to simplicity and low cost, resulted to be UA-SLE performed with $0.1 \mathrm{~g}$ of defatted sample and $15 \mathrm{~mL}$ of solvent $(1 \mathrm{~mL}$ methanol $/ 1 \mathrm{~mL}$ water $/ 8 \mathrm{~mL}$ methanol $0.1 \%$ formic acid $/ 5 \mathrm{~mL}$ acetonitrile) [7].

A number of studies focused on the extraction of phenolic compounds from $C$. avellana byproducts like skins and shells have been reported $[18,55,80]$. The skin that envelops hazelnut kernels is usually removed after roasting process, representing a phenolic-rich by-product.

Different extraction solvents (methanol, acidified methanol, ethanol, acidified ethanol, and acetone/water) and protocols (cold solvent-assisted extraction and semi-automated Soxhlet extraction) have been evaluated in different papers to extract antioxidants from hazelnut shells and pellicular wastes coming from the industrial pericarp removal during the processing of hazelnut kernels. To optimize parameters like solvent and extraction time and to evaluate their role in phenolics extraction from the skin, in 2007 Stevigny et al. applied an experimental design approach. Solvent composition (X1) and extraction time (X2) were chosen as independent variables. Response surface methodology was then applied to predict the best conditions for standardized extraction of phenolics [80]. A similar approach applying experimental design to extraction parameters was followed by Yuan et al. in 2018. Preliminary optimization showed that a high recovery of phenolics could be achieved with shell particle size less than $0.5 \mathrm{~mm}$ when extracted with acetone at $50^{\circ} \mathrm{C}$. Response surface experiments showed that a $10 \mathrm{~g} / \mathrm{L}$ liquid to solid ratio, $58 \%$ acetone, and 12-h extraction time yielded the highest amount of phenolics [18].

Other extraction approaches, like alkaline hydrolysis [55] and ultrasound-assisted extraction compared with microwave-assisted extraction and supercritical dioxide extraction, were also explored and applied to skin and shells byproducts of hazelnut production [81].

Studies on extraction methods to optimize phenolics recovery from leaves are not so easy to find. Only one paper explored this item, with the aim to underline the chemical composition of extracts of the leaves obtained by infusion usually used in folk med- 
- Table 1 Amount of metabolites occurring in C. avellana.

\begin{tabular}{|c|c|c|c|c|c|}
\hline & Compounds & Part & Concentration (mg/kg) & Methods & References \\
\hline 1 & p-hydroxybenzoic acid & kernels & $26.5 \pm 1.9$ & LC-MS & [51] \\
\hline 4 & gallic acid & $\begin{array}{l}\text { shells } \\
\text { kernels }\end{array}$ & $\begin{array}{r}62.1 \pm 4.3 \\
4.4 \pm 0.7\end{array}$ & HPLC-DAD & {$[18,23]$} \\
\hline 7 & protocatechuic acid & shells & $22.1 \pm 1.8$ & HPLC-DAD & [23] \\
\hline 14 & m-hydroxycinnamic acid & kernels & $86.1 \pm 2.6$ & LC-MS & [51] \\
\hline 21 & 3-caffeoylquinic acid & leaves & $1160.0 \pm 0.0$ & HPLC-DAD & [13] \\
\hline 23 & 5-caffeoylquinic acid & leaves & $1110.0 \pm 0.0$ & HPLC-DAD & [13] \\
\hline 24 & p-coumaroyltartaric acid & leaves & $30.0 \pm 0.0$ & HPLC-DAD & [13] \\
\hline 25 & caffeoyltartaric acid & leaves & $20.0 \pm 0.0$ & HPLC-DAD & [13] \\
\hline 26 & rosmarinic acid & leaves & $9050.0 \pm 0.1$ & LC-MS & {$[22]$} \\
\hline 30 & quercetin & shells & $40.4 \pm 3.6$ & HPLC-DAD & [18] \\
\hline 32 & quercetin 3-rhamnoside & $\begin{array}{l}\text { kernels } \\
\text { shells } \\
\text { flower } \\
\text { leaves }\end{array}$ & $\begin{array}{r}4.9 \pm 0.0 \\
20.9 \pm 1.8 \\
65.3 \pm 0.9 \\
16940.0 \pm 0.3\end{array}$ & $\begin{array}{l}\text { LC-MS } \\
\text { HPLC-DAD } \\
\text { LC-MS }\end{array}$ & {$[9,18,25]$} \\
\hline 33 & myricetin 3-rhamnoside & $\begin{array}{l}\text { kernels } \\
\text { leaves } \\
\text { bark }\end{array}$ & $\begin{array}{r}1.2 \pm 0.0 \\
37700.0 \pm 0.9 \\
2420.0 \pm 0.1\end{array}$ & LC-MS & {$[22,25]$} \\
\hline 34 & kaempferol 3-rhamnoside & $\begin{array}{l}\text { flowers } \\
\text { leaves } \\
\text { bark }\end{array}$ & $\begin{array}{r}12.0 \pm 0.4 \\
1460.0 \pm 0.2 \\
4470.0 \pm 0.1\end{array}$ & LC-MS & {$[9,22]$} \\
\hline 35 & quercetin 3-glucoside & $\begin{array}{l}\text { flowers } \\
\text { leaves } \\
\text { bark }\end{array}$ & $\begin{aligned} 13.6 & \pm 0.2 \\
3490.0 & \pm 0.04 \\
2310.0 & \pm 0.1\end{aligned}$ & LC-MS & {$[9,22]$} \\
\hline 39 & quercetin 3-galactosyl-( $1 \rightarrow 2)$-glucoside & flowers & $493.3 \pm 2.6$ & LC-MS & [9] \\
\hline 40 & kaempferol 3-glucosyl-( $(\rightarrow$ 2)-glucoside & flowers & $654.7 \pm 3.6$ & LC-MS & [9] \\
\hline 41 & kaempferol 3-(cis-p-coumaroyl)-rhamnoside & $\begin{array}{l}\text { shells } \\
\text { flowers }\end{array}$ & $\begin{array}{c}98.0 \pm 1.0 \\
533.0 \pm 11.5\end{array}$ & LC-MS & {$[9,20]$} \\
\hline 42 & kaempferol 3-(trans-p-coumaroyl)-rhamnoside & $\begin{array}{l}\text { shells } \\
\text { flowers }\end{array}$ & $\begin{array}{r}64.0 \pm 1.4 \\
177.3 \pm 3.8\end{array}$ & LC-MS & {$[9,20]$} \\
\hline 48 & phloretin-2'-O-glucoside & kernels & $10.6 \pm 0.0$ & LC-MS & {$[25]$} \\
\hline 49 & catechin & $\begin{array}{l}\text { shells } \\
\text { kernels }\end{array}$ & $\begin{array}{r}176.4 \pm 6.4 \\
12.0 \pm 0.0\end{array}$ & HPLC-DAD & {$[18,51]$} \\
\hline 50 & epicatechin & $\begin{array}{l}\text { shells } \\
\text { kernels }\end{array}$ & $\begin{array}{r}17.1 \pm 1.5 \\
2.0 \pm 0.0\end{array}$ & HPLC-DAD & {$[18,77]$} \\
\hline 51 & epigallocatechin & kernels & $28.0 \pm 0.0$ & & [77] \\
\hline 53 & epicatechin gallate & $\begin{array}{l}\text { shells } \\
\text { kernels }\end{array}$ & $\begin{array}{l}63.2 \pm 5.7 \\
11.0 \pm 0.0\end{array}$ & HPLC-DAD & {$[18,51]$} \\
\hline 55 & gallocatechin gallate & kernels & $12.0 \pm 0.0$ & & [77] \\
\hline 62 & hirsutenone & leaves & $330.0 \pm 0.0$ & LC-MS & [22] \\
\hline 88 & carpinontriol B & shells & $223.0 \pm 1.4$ & LC-MS & {$[20]$} \\
\hline 89 & giffonin V & shells & $248.0 \pm 3.0$ & LC-MS & [20] \\
\hline 90 & alnusone & flowers & $510.7 \pm 3.4$ & LC-MS & [9] \\
\hline 91 & erythro-(7S,8R)-guaiacylglycerol- $\beta-0-4^{\prime}$-dihydroconiferyl alcohol & shells & $833.0 \pm 1.8$ & LC-MS & [20] \\
\hline 92 & erythro-(7S,8R)-guaiacylglycerol- $\beta$-coniferyl aldehyde ether & shells & $332.0 \pm 1.0$ & LC-MS & [20] \\
\hline 93 & erythro-(7R,8S)-guaiacylglycerol- $\beta$-O-4'-dihydroconiferyl alcohol & shells & $553.0 \pm 5.2$ & LC-MS & [20] \\
\hline 96 & ficusal & shells & $722.0 \pm 5.4$ & LC-MS & {$[20]$} \\
\hline 98 & dihydrodehydrodiconiferyl alcohol & shells & $573.0 \pm 8.7$ & LC-MS & [20] \\
\hline 99 & balanophonin & shells & $118.0 \pm 1.6$ & LC-MS & {$[20]$} \\
\hline
\end{tabular}


icine; extracts were obtained by different procedures and the occurrence of phytochemicals was then investigated. Each extraction method presented its own advantages and disadvantages, and particularly in this study, extracts were obtained by "ecofriendly" procedures in order to explore their potential use as functional ingredients for pharmaceutical and cosmetic formulations [63].

\section{HPLC and HPLC/MS Analysis}

Although kernels in past were studied mainly for their lipid and volatile content, in 2006 Kornsteiner carried out a study aimed at assessing the content of tocopherols and carotenoids in the unsaponifiable fraction as well as the amount of phenols in 10 different types of nuts by HPLC [82]. After this work, other authors evaluated by HPLC a limited number of phenolic compounds in kernels [16,33]. Recently, in a study concerning polyphenols analysis in kernels, 5 phenolic acids, namely gallic acid (4), caffeic acid (11), p-coumaric acid (10), ferulic acid (12), and sinapic acid (13), were identified and quantified (both free and esterified) by HPLC-DAD, with DAD set at 280 and $320 \mathrm{~nm}$ [3]. In the same paper byproducts (skin, hard shell, green leafy cover, and tree leaf) were evaluated too for determining the contents of the 5 phenolic compounds.

In 2010 Jakopic et al. explored phenolics in kernel of 20 cultivars by HPLC-MS. Twenty-three compounds from different phenolic groups were detected, and 15 of them were identified. No individual compound was quantified. Total phenol concentrations ranged from 70 to $478 \mathrm{mg}$ gallic acid equivalents per kg hazelnut kernels. A high level of total phenols was observed in the "Tonda Gentile delle Langhe" and "Lewis" cultivars, followed by "Corabel," "Fertile de Coutard," "Daria," and "Tonda Gentile Romana" [25]. A similar approach, with LC-MS used for identification of phenolic compounds, was published in the same year [59]. HPLC-MS identification of individual phenolics confirmed the presence of 2 hydroxybenzoic acids, namely gallic acid (4) and protocatechuic acid (7), quercetin-3-rhamnoside (32), and myricetin 3-rhamnoside (33), 1 dihydrochalcone, phloretin-2-O-glucoside (48), catechin (49), epicatechin (50), 2 procyanidin dimers, and 3 procyanidin trimers, and flavonols like quercetin pentoside.

In 2014, 29 hazelnut cultivars were evaluated and a detailed structural characterization of kernel polyphenols was performed, confirming the presence of protocatechuic acid (7), flavan-3-ols such as catechin (49), procyanidin B2 (59), 6 procyanidin oligomers, flavonols, and 1 dihydrochalcone in all the analyzed cultivars. The qualitative analysis was carried out by LC-ESI-IT-MS while the content of the single polyphenolic compounds was determined by HPLC-UV. The analysis of a larger number of cultivars of different geographic origins validated the method and the results highlighted that a molecular profiling strategy based on phenolics is able to discriminate hazelnut cultivars [10].

In the same year, Slatnar published a paper reporting the phenolic content of kernels, bagasse pellets (residue of oil pressing) and oils from 5 different cultivars. Phenolic compounds were identified by LC-MS. The main polyphenolic subclass comprised mono- and oligomeric flavan-3-ols, which accounted between 34.2 and $58.3 \%$ of the total phenolics detected in kernels and between 36.7 and $48.6 \%$ in pellets. In hazelnut oils 4 compounds have been detected, their content levels ranged from 0.97 to $0.01 \mu \mathrm{g} / \mathrm{g}[51]$.

UHPLC was also applied for the analysis of phenolic compounds in hazelnut kernels. Gültekin-Ozgüven provided a rapid method for the quali-quantitative analysis of phenolics by UHPLC-MS by using an instrument equipped with a Triple Quadrupole analyzer [83]. By combination of UHPLC and short length columns, higher speed was achieved compared to a conventional HPLC. Gallic acid (4), syringic acid (6), protocatechuic acid (7), p-coumaric acid (10), caffeic acid (11), ferulic acid (12), o-coumaric acid (15), 3caffeoylquinic acid (21), rosmarinic acid (26), rutin (38), catechin (49), epicatechin $(50)$, and kaempferol derivatives $(34,36)$ were identified and determined in hazelnut extracts. Recently, Fanali et al. [7] analyzed hazelnut kernels for the determination of individual phenolic compounds by HPLC coupled with DAD and MS detections. Six phenolic compounds belonging to different phenolic classes were used as standard. Then the method was fully validated. A good linearity was obtained giving R2 no lower than 0.997. Recently a qualitative analytical approach based on HPLC coupled to electrospray ionization, multiple-stage linear ion-trap and Orbitrap HRMS (LC-ESI-LTQOrbitrap-MS/MSn) of the fresh and roasted kernel of the Italian cultivar Tonda di Giffoni evidenced 11 phenolic compounds: the flavonoid O-glycosides quercetin 3-rhamnoside (32), myricetin 3-rhamnoside (33), quercetin 3-glucoside (35), kaempferol 3-glucoside (36), kaempferol 3-(4"'-cis-p-coumaroyl)-rhamnoside (41), kaempferol 3-(4"'trans-p-coumaroyl)-rhamnoside (42), along with ellagic acid (56), the diarylheptanoid derivatives giffonin Q (77), giffonin $M$ (82), giffonin $P(85)$, and carpinontriol $B(88)$ [27].

In recent years, a big interest has been directed to hazelnut byproducts, like shells and skins. In 2017 Masullo et al. explored the phenolic composition of the shells of the Italian cultivar Tonda di Giffoni. In order to perform the quantitative determination of the main compounds of the methanol extract, an analytical method based on LC-MS with ESI and triple quadrupole mass analyzer (QqQ), using multiple reaction monitoring (MRM) scan mode, was developed and validated. This analytical approach showed erythro-(7S,8R)-guaiacylglycerol- $\beta$-O-4'-dihydroconiferyl alcohol (90), ficusal (95), and dihydrodehydrodiconiferyl alcohol (97) as the main compounds. The quantitative results highlighted that the main compounds occurred in the extract in concentration ranging from 6.4 to $83.3(\mathrm{mg} / 100 \mathrm{~g})$ [20]. The chemical characterization of shell and skin of another Italian hazelnut Tonda Gentile Romana was carried out using analytical pyrolysis in the presence of hexamethyldisilazane followed by GC-MS analysis and HPLC-DAD by Mattonai et al. [31]. High concentrations of catechin (49), epicatechin (50) and procyanidins were found in the skin.

The polyphenolic profile of fresh and roasted hazelnut skins was studied by HPLC-DAD and LC-MS. A specific study on phenolic compounds in roasted hazelnut skins evidenced the presence of flavan-3-ols mainly constituted by B-type proanthocyanidins (up to degree of polymerization to 9). The polyphenolic profile of hazelnut skins was compared with the polyphenolic profile of roasted skins obtained from different nuts [32]. The proanthocyanidins profile was more accurately investigated in 2016 by Picci- 
nelli et al. [34]. In 2018 a study that evaluated the change in phenolics during ripening in skins and kernels was carried out [84].

In 2005 Amaral et al. performed an investigation aimed at defining the profile of polyphenols in leaves of $C$. avellana. Thus phenolic compounds of hazelnut leaves of 10 different cultivars developed under the same cultural, geographical, geological, and climatic conditions were analyzed by HPLC-DAD and HPLC-DADESI-MS/MS. Eight phenolic compounds, 3-caffeoylquinic acid (21), 5-caffeoylquinic acid (23), p-coumaroyltartaric acid (24), caffeoyltartaric acid (25), quercetin 3-rhamnoside (32), myricetin 3-rhamnoside (33), kaempferol 3-rhamnoside (34), and quercetin 3-glucoside (35) were identified and quantified. All of the analyzed samples showed a similar phenolic profile, in which quercetin 3-rhamnoside (32) and myricetin 3-rhamnoside (33) were the major compounds [26]. A further study on leaves of C. avellana was published in 2007. Aqueous extracts of leaves of different hazel cultivars (M. Bollwiller, Fertille de Coutard, and Daviana) were analyzed by reversed-phase HPLC-DAD for the characterization of their phenolic composition [21]. Phenolic compounds of leaves were quantitatively evaluated, with their geoclimatic and seasonal variations [13]. In this research, a seasonal pattern variation study was performed, comprising the screening of the phenolic composition of 4 cultivars under the same agricultural, geographical, and climatic conditions. A seasonal pattern was observed consisting of an increase of the total phenolic content from May to July, a considerable decrease in August, and a new increase in September. In all cultivars, the highest content of phenolics was achieved in July. Multivariate statistical analysis was also applied.

Leaves and barks were studied for their phenolic content in 2013 by Riethmüller et al. [22]. UV spectroscopic data, obtained by LC-DAD, accurate molecular mass and formula, acquired by LC and ESI with time-of-flight MS and fragmentation pattern, given by LC-ESI/MS/MS analyses were carried out. Then a specific MRM MS/MS method was developed and validated for the following phenolic compounds: caffeic acid (11), rosmarinic acid (26), quercetin 3-rhamnoside (32), myricetin 3-rhamnoside (33), kaempferol 3-rhamnoside (34), hirsutenone (62), 1,7-bis-(4-hydroxyphenyl)-4,6-heptadien-3-one (63), 5-hydroxy-1-(4-hydroxyphenyl)7-(3,4-dihydroxyphenyl)-heptan-3-one (64), 5-hydroxy-1-(3,4-dihydroxyphenyl)-7-(4-hydroxyphenyl)-heptan-3-one (65), along with kaempferol-di(desoxyhexoside), quercetin 3-hexoside, myricetin 3-hexoside, 1,7-bis-(4hydroxyphenyl)-4-hepten-3-onehexoside, 1,7-bis-(4-hydroxyphenyl)-4,6-heptadien-3-one-hexoside, hirsutenone-hexoside. Quantitation of the compounds was performed by LC-ESI-MS/MS [22].

An analytical method based on LC-ESI-MS was developed for male flowers. An initial LC-MS qualitative analysis of the methanolic extract of the male flowers of $C$. avellana was performed showing the occurrence of 12 phenolic compounds. Among them 11 compounds were quantified: quercetin 3-rhamnoside (32), kaempferol 3-rhamnoside (34), quercetin 3-glucoside (35), quercetin 3-galactosyl-( $1 \rightarrow 2)$-glucoside (39), kaempferol 3-glucosyl( $1 \rightarrow 2$ )-glucoside (40), kaempferol 3-(4'"-cis-p-coumaroyl)-rhamnoside (41), giffonin Q (77), giffonin R (78), giffonin S (79), giffonin I (80) and alnusone (90). The quantitative determination of the main compounds was carried out by LC-ESI(QqQ)MS, using the Multiple Reaction Monitoring (MRM) [9].

\section{NMR and Genomics Combined with Multivariate Data Analysis}

Traditional methods to identify hazelnut cultivars are based on phenotypic observations, but unfortunately, they are affected by environmental and developmental factors. DNA-typing methods such as random amplification of polymorphic DNA, inter-simple sequence repeat, restriction fragment length polymorphism, amplification refractory mutation system, and simple sequence repeat are known to be useful for revealing genetic polymorphisms among different cultivars and accurately identifying hazelnut cultivars but are laborious and time-consuming $[5,85]$. Most of these methods are not specific but give a total fingerprint that can be submitted to statistical evaluation by multivariate data analysis able to identify similitudes and differences in samples and variable involved in their differentiation. Principal component analysis (PCA) and partial least square (PLS) are the multivariate models mainly applied to the evaluation of these problems, together with hierarchical clustering analysis. An analytical technique that was an alternative tool to obtain very informative and robust fingerprint is NMR. Several authors have used principally ${ }^{1} \mathrm{H}$ NMR spectroscopy combined to chemometric tools to discriminate hazelnut samples. In 2014 high-resolution ${ }^{1} \mathrm{H}$ NMR spectroscopy was performed on 3 Italian hazelnut cultivars, Tonda di Giffoni, Mortarella, and Tonda Gentile Romana, to characterize their metabolite profile. The hazelnuts were grown in the same pedoclimatic conditions and in the same geographical area. Metabolites belonging to different chemical classes (amino acids, organic acids, carbohydrates, lipids, and miscellaneous compounds) were identified and quantified. In addition, the fingerprint combined with PCA was used to discriminate the classes [86]. In the same year ${ }^{1} \mathrm{H}$ NMR analyses were carried out on polar extracts of "Tonda gentile trilobata" and other cultivars and the data were analyzed by multivariate statistical methods [87]. In 2018 NMR fingerprint combined with multivariate data analysis was applied to discriminate the geographical origin of hazelnuts. The work addressed for the first time the untargeted NMR spectroscopic analysis of a large quantity of hazelnut samples from 5 different Eurasian countries. The data analysis generated a model predicting the origin of samples from a test set with a high accuracy [88]. All the previous described papers concern kernels [89], while Cerulli et al. in 2018 explored the metabolome of leaves of $C$. avellana by ${ }^{1} \mathrm{H}$ NMR combined with multivariate data analysis (PCA and PLS-discriminant analysis) and to date it remains the only paper describing a nontargeted metabolomics approach on a C. avellana part different from hazelnut [63]. Metabolites belonging to different classes, different amino acids and sugars (primary metabolites) occurring with quercetin 3-rhamnoside (32), myricetin 3-rhamnoside (33), kaempferol 3-rhamnoside (34), kaempferol 3-O-(4"'-trans-p-coumaroyl) rhamnopyranoside (42), giffonin A (67), giffonin B (68), giffonin $C(69)$, giffonin $D(70)$, giffonin $E(71)$, giffonin $F(72)$, giffonin $G(73)$, giffonin $H(74)$, giffonin I (80), giffonin $K(76)$, giffo$\operatorname{nin} L(81)$, giffonin $M(82)$, giffonin $N(83)$, giffonin $O(84)$, giffo$\operatorname{nin} \mathrm{P}(85)$, giffonin $\mathrm{T}(86)$, giffonin $U$ (87), and carpinontriol $B(88)$ were identified and used for samples classification. 


\section{Conclusion}

This review highlights how in the recent years, together with the ongoing research carried out on hazelnut kernels, a growing interest has been addressed to the hazelnut byproducts, represented by hazelnut skins, hazelnut hard shells, and hazelnut green leafy covers as well as hazelnut tree leaves. A lot of analytical works have been carried out to define the quali-quantitative profiles of the different parts of $C$. avellana.

Phenolics are the main specialized metabolites. Besides phenolic acids and flavonoids, recent investigation highlighted the presence of diarylheptanoids, less common specialized metabolites, characterized by a highly hydroxylated cyclic diarylheptanoid skeleton.

Extracts obtained from the different parts of C. avellana along with pure compounds have shown several biological activities including antioxidant, antiproliferative, antimicrobial along with neuroprotective effects. Thus, the present work contributes to highlight $C$. avellana as a source of bioactive principles, but further studies, cohort or case-control studies, are required to address the health benefits of $C$. avellana extracts and their possible use as food supplements.

Concerning taxanes isolated from in vitro cell cultures of different explants of hazelnut and also reported in hazelnut leaves, at the moment there are no scientific evidences to sustain $C$. avellana as a more abundant and available source of these molecules than yew. Therefore, considering the importance of the matter, additional investigations specifically addressed to highlight the occurrence of taxanes in hazelnut and related byproducts are needed.

\section{Conflict of Interest}

The authors declare that they have no conflict of interest.

\section{References}

[1] Alasalvar C, Bolling BW. Review of nut phytochemicals, fat-soluble bioactives, antioxidant components and health effects. $\mathrm{Br}$ J Nutr 2015; 113: S68-S78

[2] Alasalvar C, Karamac M, Amarowicz R, Shahidi F. Antioxidant and antiradical activities in extracts of hazelnut kernel (Corylus avellana L.) and hazelnut green leafy cover. J Agric Food Chem 2006; 54: 4826-4832

[3] Shahidi F, Alasalvar C, Liyana-Pathirana CM. Antioxidant phytochemicals in hazelnut kernel (Corylus avellana L.) and hazelnut byproducts. J Agric Food Chem 2007; 55: 1212-1220

[4] Alasalvar C, Shahidi F. Natural antioxidants in tree nuts. Eur J Lipid Sci Tech 2009; 111: 1056-1062

[5] Marinoni DT, Valentini N, Portis E, Acquadro A, Beltramo C, Mehlenbacher SA, Mockler TC, Rowley ER, Botta R. High density SNP mapping and QTL analysis for time of leaf budburst in Corylus avellana L. PLoS One 2018; 13: e0195408/1-e0195408/24

[6] Masullo M, Cerulli A, Olas B, Pizza C, Piacente S. Giffonins A-I, antioxidant cyclized diarylheptanoids from the leaves of the hazelnut tree (Corylus avellana), source of the Italian PGI Product "Nocciola di Giffoni". J Nat Prod 2015; 78: 17-25

[7] Fanali C, Tripodo G, Russo M, Della Posta S, Pasqualetti V, De Gara L. Effect of solvent on the extraction of phenolic compounds and antioxidant capacity of hazelnut kernel. Electrophoresis 2018; 39: 1683-1691
[8] Cerulli A, Lauro G, Masullo M, Cantone V, Olas B, Kontek B, Nazzaro F, Bifulco G, Piacente S. Cyclic diarylheptanoids from Corylus avellana green leafy covers: determination of their absolute configurations and evaluation of their antioxidant and antimicrobial activities. J Nat Prod 2017; 80: 1703-1713

[9] Masullo M, Mari A, Cerulli A, Bottone A, Kontek B, Olas B, Pizza C, Piacente S. Quali-quantitative analysis of the phenolic fraction of the flowers of Corylus avellana, source of the Italian PGI product "Nocciola di Giffoni": isolation of antioxidant diarylheptanoids. Phytochemistry (Elsevier) 2016; 130: 273-281

[10] Ciarmiello LF, Mazzeo MF, Minasi P, Peluso A, De Luca A, Piccirillo P, Siciliano RA, Carbone V. Analysis of different European hazelnut (Corylus avellana L.) cultivars: authentication, phenotypic features, and phenolic profiles. J Agric Food Chem 2014; 62: 6236-6246

[11] Gorji N, Moeini R, Memariani Z. Almond, hazelnut and walnut, three nuts for neuroprotection in Alzheimer's disease: a neuropharmacological review of their bioactive constituents. Pharmacol Res 2018; 129: 115-127

[12] Akbarzadeh T, Sabourian R, Saeedi M, Rezaeizadeh H, Khanavi M, Ardekani MRS. Liver tonics: review of plants used in Iranian traditional medicine. Asian Pac J Trop Biomed 2015; 5: 170-181

[13] Amaral JS, Valentao P, Andrade PB, Martins RC, Seabra RM. Phenolic composition of hazelnut leaves: influence of cultivar, geographical origin and ripening stage. Sci Hortic (Amsterdam, Neth) 2010; 126: 306-313

[14] Tunon H, Olavsdotter C, Bohlin L. Evaluation of anti-inflammatory activity of some Swedish medicinal plants. Inhibition of prostaglandin biosynthesis and PAF-induced exocytosis. J Ethnopharmacol 1995; 48: 61-76

[15] Masullo M, Montoro P, Mari A, Pizza C, Piacente S. Medicinal plants in the treatment of women's disorders: analytical strategies to assure quality, safety and efficacy. J Pharm Biomed Anal 2015; 113: 189-211

[16] Prosperini S, Ghirardello D, Scursatone B, Gerbi V, Zeppa G. Identification of soluble phenolic acids hazelnut (Corylus avellana L.) kernel. Acta Hortic 2009; 845: 677-680

[17] Pelvan E, Olgun EO, Karadag A, Alasalvar C. Phenolic profiles and antioxidant activity of Turkish Tombul hazelnut samples (natural, roasted, and roasted hazelnut skin). Food Chem 2018; 244: 102-108

[18] Yuan B, Lu M, Eskridge KM, Isom LD, Hanna MA. Extraction, identification, and quantification of antioxidant phenolics from hazelnut (Corylus avellana L.) shells. Food Chem 2018; 244: 7-15

[19] Esposito T, Sansone F, Franceschelli S, Del Gaudio P, Picerno P, Aquino RP, Mencherini T. Hazelnut (Corylus avellana L.) shells extract: phenolic composition, antioxidant effect and cytotoxic activity on human cancer cell lines. Int J Mol Sci 2017; 18: 392/1-392/12

[20] Masullo M, Cerulli A, Mari A, de Souza Santos CC, Pizza C, Piacente S. LC-MS profiling highlights hazelnut (Nocciola di Giffoni PGI) shells as a byproduct rich in antioxidant phenolics. Food Res Int 2017; 101: 180187

[21] Oliveira I, Sousa A, Valentao P, Andrade PB, Ferreira ICFR, Ferreres F, Bento A, Seabra R, Estevinho L, Pereira JA. Hazel (Corylus avellana L.) leaves as source of antimicrobial and antioxidative compounds. Food Chem 2007; 105: 1018-1025

[22] Riethmueller E, Alberti A, Toth G, Beni S, Ortolano F, Kery A. Characterisation of diarylheptanoid- and flavonoid-type phenolics in Corylus avellana L. Leaves and bark. Phytochem Anal 2013; 24: 493-503

[23] Solar A, Veberic R, Bacchetta L, Botta R, Drogoudi P, Metzidakis I, Rovira $M$, Sarraquigne JP, Silva AP. Phenolic characterization of some hazelnut cultivars from different European germplasm collections. Acta Hortic 2009; 845: 613-618

[24] Del Rio D, Calani L, Dall Asta M, Brighenti F. Polyphenolic composition of hazelnut skin. J Agric Food Chem 2011; 59: 9935-9941

[25] Jakopic J, Petkovsek MM, Likozar A, Solar A, Stampar F, Veberic R. HPLC-MS identification of phenols in hazelnut (Corylus avellana L.) kernels. Food Chem 2010; 124: 1100-1106 
[26] Amaral JS, Ferreres F, Andrade PB, Valentao P, Pinheiro C, Santos A, Seabra R. Phenolic profile of hazelnut (Corylus avellana L.) leaves [of] cultivars grown in Portugal. Nat Prod Res 2005; 19: 157-163

[27] Cerulli A, Napolitano A, Masullo M, Pizza C, Piacente S. LC-ESI/ LTQOrbitrap/MS/MS analysis reveals diarylheptanoids and flavonol Oglycosides in fresh and roasted hazelnut (Corylus avellana cultivar "Tonda di Giffoni”). Nat Prod Commun 2018; 13: 1123-1126

[28] Peev Cl, Vlase L, Antal DS, Dehelean CA, Szabadai Z. Determination of some polyphenolic compounds in buds of Alnus and Corylus species by HPLC. Chem Nat Compd 2007; 43: 259-262

[29] Strack D, Meurer B, Wray V, Grotjahn L, Austenfeld FA, Wiermann R. Quercetin 3-glucosylgalactoside from pollen of Corylus avellana. Phytochemistry 1984; 23: 2970-2971

[30] Masullo M, Cantone V, Cerulli A, Lauro G, Messano F, Russo GL, Pizza C, Bifulco G, Piacente S. Giffonins J-P, highly hydroxylated cyclized diarylheptanoids from the leaves of Corylus avellana cultivar "Tonda di Giffoni". J Nat Prod 2015; 78: 2975-2982

[31] Mattonai M, Licursi D, Antonetti C, Raspolli Galletti AM, Ribechini E. Py-GC/MS and HPLC-DAD characterization of hazelnut shell and cuticle: insights into possible re-evaluation of waste biomass. J Anal Appl Pyrolysis 2017; 127: 321-328

[32] Monagas M, Garrido I, Lebron-Aguilar R, Gomez-Cordoves MC, Rybarczyk A, Amarowicz R, Bartolome B. Comparative flavan-3-ol profile and antioxidant capacity of roasted peanut, hazelnut, and almond skins. J Agric Food Chem 2009; 57: 10590-10599

[33] Ghirardello D, Prosperini S, Zeppa G, Gerbi V. Phenolic acid profile and antioxidant capacity of hazelnut (Corylus avellana L.) kernels in different solvent systems. J Food Nutr Res 2010; 49: 195-205

[34] Piccinelli AL, Pagano I, Esposito T, Mencherini T, Porta A, Petrone AM, Gazzerro P, Picerno P, Sansone F, Rastrelli L, Aquino RP. HRMS profile of a hazelnut skin proanthocyanidin-rich fraction with antioxidant and antiCandida albicans activities. J Agric Food Chem 2016; 64: 585-595

[35] Gallego A, Meton I, Baanante IV, Ouazzani J, Adelin E, Palazon J, Bonfill M, Moyano E. Viability-reducing activity of Coryllus avellana L. extracts against human cancer cell lines. Biomed Pharmacother 2017; 89: 565572

[36] Daquino C, Rescifina A, Spatafora C, Tringali C. Biomimetic synthesis of natural and "Unnatural" lignans by oxidative coupling of caffeic esters. Eur J Org Chem 2009; 36: 6289-6300

[37] Bestoso F, Ottaggio L, Armirotti A, Balbi A, Damonte G, Degan P, Mazzei M, Cavalli F, Ledda B, Miele M. In vitro cell cultures obtained from different explants of Corylus avellana produce Taxol and taxanes. BMC Biotechnol 2006; 6: 45

[38] Ottaggio L, Bestoso F, Armirotti A, Balbi A, Damonte G, Mazzei M, Sancandi M, Miele M. Taxanes from shells and leaves of Corylus avellana. J Nat Prod 2008; 71: 58-60

[39] Hoffman A, Shahidi F. Paclitaxel and other taxanes in hazelnut. J Funct Foods 2008; 1 : 33-37

[40] Phuc DTH, Popovich DG. Screening for paclitaxel and other taxanes in kernel and shell of Corylus avellana (Hazelnut). J Pharmacogn Phytochem 2017; 6: 247-254

[41] Alasalvar C, Shahidi F, Cadwallader KR. Comparison of natural and roasted Turkish Tombul hazelnut (Corylus avellana L.) volatiles and flavor by DHA/GC/MS and descriptive sensory analysis. I Agric Food Chem 2003; 51: 5067-5072

[42] Cordero C, Liberto E, Bicchi C, Rubiolo P, Schieberle P, Reichenbach SE, Tao Q. Profiling food volatiles by comprehensive two-dimensional gas chromatography coupled with mass spectrometry: advanced fingerprinting approaches for comparative analysis of the volatile fraction of roasted hazelnuts (Corylus avellana L.) from different origins. J Chromatogr A 2010; 1217: 5848-5858

[43] Marzocchi S, Pasini F, Verardo V, Ciemniewska-Zytkiewicz H, Caboni MF, Romani S. Effects of different roasting conditions on physical-chemical properties of Polish hazelnuts (Corylus avellana L. var. Katalonski). LWTFood Sci Technol 2017; 77: 440-448

[44] Shahidi F, Ambigaipalan P. Phenolics and polyphenolics in foods, beverages and spices: antioxidant activity and health effects - a review. J Funct Foods 2015; 18: 820-897

[45] Costa C, Tsatsakis A, Mamoulakis C, Teodoro M, Briguglio G, Caruso E, Tsoukalas D, Margina D, Dardiotis E, Kouretas D, Fenga C. Current evidence on the effect of dietary polyphenols intake on chronic diseases. Food Chem Toxicol 2017; 110: 286-299

[46] Shahidi F, Zhong Y. Measurement of antioxidant activity. J Funct Foods 2015; 18: 757-781

[47] Kowalska I, Jedrejek D, Ciesla L, Pecio L, Masullo M, Piacente S, Oleszek W, Stochmal A. Isolation, chemical and free radical scavenging characterization of phenolics from Trifolium scabrum L. aerial parts. J Agric Food Chem 2013; 61: 4417-4423

[48] Bolling BW, Chen CYO, McKay DL, Blumberg JB. Tree nut phytochemicals: composition, antioxidant capacity, bioactivity, impact factors. A systematic review of almonds, Brazils, cashews, hazelnuts, macadamias, pecans, pine nuts, pistachios and walnuts. Nutr Res Rev 2011; 24: 244275

[49] Binello A, Giorgis M, Cena C, Cravotto G, Rotolo L, Oliveri P, Malegori C, Cavallero MC, Buso S, Casale M. Chemical modifications of Tonda Gentile Trilobata hazelnut and derived processing products under different infrared and hot-air roasting conditions: a combined analytical study. J Sci Food Agr 2018; 98: 4561-4569

[50] Ozcan MM, Al Juhaimi F, Uslu N. The effect of heat treatment on phenolic compounds and fatty acid composition of Brazilian nut and hazelnut. J Food Sci Tech Mys 2018; 55: 376-380

[51] Slatnar A, Mikulic-Petkovsek M, Stampar F, Veberic R, Solar A. HPLC-MS identification and quantification of phenolic compounds in hazelnut kernels, oil and bagasse pellets. Food Res Int 2014; 64: 783-789

[52] Pelvan E, Alasalvar C, Uzman S. Effects of roasting on the antioxidant status and phenolic profiles of commercial Turkish hazelnut varieties (Corylus avellana L.). J Agric Food Chem 2012; 60: 1218-1223

[53] Mollica A, Zengin G, Stefanucci A, Ferrante C, Menghini L, Orlando G, Brunetti L, Locatelli M, Dimmito MP, Novellino E, Wakeel OK, Ogundeji MO, Onaolapo AY, Onaolapo OJ. Nutraceutical potential of Corylus avellana daily supplements for obesity and related dysmetabolism. J Funct Foods 2018; 47: 562-574

[54] Ghirardello D, Bertolino M, Belviso S, Dal Bello B, Giordano M, Rolle L, Gerbi V, Antonucci M, Spigolon N, Zeppa G. Phenolic composition, antioxidant capacity and hexanal content of hazelnuts (Corylus avellana L.) as affected by different storage conditions. Postharvest Biol Tec 2016; 112: 95-104

[55] Tas NG, Gokmen V. Bioactive compounds in different hazelnut varieties and their skins. J Food Compos Anal 2015; 43: 203-208

[56] Kalkan F, Vanga SK, Gariepy Y, Raghavan V. Effect of MW-assisted roasting on nutritional and chemical properties of hazelnuts. Food Nutr Res 2015; 59: 28916

[57] Oliveira I, Sousa A, Morais JS, Ferreira ICFR, Bento A, Estevinho L, Pereira JA. Chemical composition, and antioxidant and antimicrobial activities of three hazelnut (Corylus avellana L.) cultivars. Food Chem Toxicol 2008; 46: $1801-1807$

[58] Li H, Parry JW. Phytochemical compositions, antioxidant properties, and colon cancer antiproliferation effects of Turkish and Oregon hazelnut. Food Nutr Sci 2011; 2: 1142-1149

[59] Schmitzer V, Slatnar A, Veberic R, Stampar F, Solar A. Roasting affects phenolic composition and antioxidative activity of hazelnuts (Corylus avellana L.). J Food Sci 2011; 76: S14-S19

[60] Kim HG, Kim GW, Oh H, Yoo SY, Kim YO, Oh MS. Influence of roasting on the antioxidant activity of small black soybean (Glycine max L. Merrill). LWT-Food Sci Technol 2011; 44: 992-998 
[61] Locatelli M, Travaglia F, Coisson JD, Martelli A, Stevigny C, Arlorio M. Total antioxidant activity of hazelnut skin (Nocciola Piemonte PGI): impact of different roasting conditions. Food Chem 2010; 119: 1647-1655

[62] Montella R, Coisson JD, Travaglia F, Locatelli M, Malfa P, Martelli A, Arlorio M. Bioactive compounds from hazelnut skin (Corylus avellana L.): effects on Lactobacillus plantarum P17630 and Lactobacillus crispatus P17631. J Funct Foods 2013; 5: 306-315

[63] Cerulli A, Masullo M, Montoro P, Hosek J, Pizza C, Piacente S. Metabolite profiling of "green" extracts of Corylus avellana leaves by ${ }^{1} \mathrm{H}$ NMR spectroscopy and multivariate statistical analysis. J Pharm Biomed Anal 2018; 160: $168-178$

[64] Ranieri R, Ciaglia E, Amodio G, Picardi P, Proto MC, Gazzerro P, Laezza C, Remondelli P, Bifulco M, Pisanti S. N6-isopentenyladenosine dual targeting of AMPK and Rab7 prenylation inhibits melanoma growth through the impairment of autophagic flux. Cell Death Differ 2018; 25: 353-367

[65] Pereira AP, Ferreira IC, Marcelino F, Valentao P, Andrade PB, Seabra R, Estevinho L, Bento A, Pereira JA. Phenolic compounds and antimicrobial activity of olive (Olea europaea L. Cv. Cobrancosa) leaves. Molecules 2007; 12: 1153-1162

[66] Proestos C, Chorianopoulos N, Nychas GJE, Komaitis M. RP-HPLC analysis of the phenolic compounds of plant extracts. Investigation of their antioxidant capacity and antimicrobial activity. J Agric Food Chem 2005; 53 : 1190-1195

[67] Amarowicz R, Dykes GA, Pegg RB. Antibacterial activity of tannin constituents from Phaseolus vulgaris, Fagoypyrum esculentum, Corylus avellana and Juglans nigra. Fitoterapia 2008; 79: 217-219

[68] Williams AR, Fryganas C, Ramsay A, Mueller-Harvey I, Thamsborg SM. Direct anthelmintic effects of condensed tannins from diverse plant sources against Ascaris suum. PLoS One 2014; 9: e97053

[69] Fogliano V, Corollaro ML, Vitaglione P, Napolitano A, Ferracane R, Travaglia F, Arlorio M, Costabile A, Klinder A, Gibson G. In vitro bioaccessibility and gut biotransformation of polyphenols present in the waterinsoluble cocoa fraction. Mol Nutr Food Res 2011; 55: S44-S55

[70] Bahaeddin Z, Yans A, Khodagholi F, Hajimehdipoor H, Sahranavard S. Hazelnut and neuroprotection: improved memory and hindered anxiety in response to intra-hippocampal A beta injection. Nutr Neurosci 2017; 20: $317-326$

[71] Blyznyuk NA, Prokopenko YS, Georgiyants VA, Tsyvunin VV. A comparative phytochemical and pharmacological analysis of the extracts from leaves of the Ukrainian flora shrubs. Visn Farm 2016; 1: 29-32

[72] Ozdal T, Capanoglu E, Altay F. A review on protein-phenolic interactions and associated changes. Food Res Int 2013; 51: 954-970

[73] Arts M], Haenen GR, Wilms LC, Beetstra SA, Heijnen CG, Voss HP, Bast A. Interactions between flavonoids and proteins: effect on the total antioxidant capacity. J Agric Food Chem 2002; 50: 1184-1187

[74] Pelitli EP, Janiak MA, Amarowicz R, Alasalvar C. Protein precipitating capacity and antioxidant activity of Turkish Tombul hazelnut phenolic extract and its fractions. Food Chem 2017; 218: 584-590

[75] Mesias M, Navarro M, Gokmen V, Morales FJ. Antiglycative effect of fruit and vegetable seed extracts: inhibition of AGE formation and carbonyltrapping abilities. J Sci Food Agr 2013; 93: 2037-2044
[76] Cristofori V, Ferramondo S, Bertazza G, Bignami C. Nut and kernel traits and chemical composition of hazelnut (Corylus avellana L.) cultivars. J Sci Food Agr 2008; 88: 1091-1098

[77] Rothwell JA, Perez-Jimenez J, Neveu V, Medina-Remon A, M'Hiri N, Garcia-Lobato P, Manach C, Knox C, Eisner R, Wishart DS, Scalbert A. Phenol-Explorer 3.0: a major update of the Phenol-Explorer database to incorporate data on the effects of food processing on polyphenol content. Database (Oxford) 2013; 2013: bat070

[78] Cevallos-Cevallos JM, Reyes-De-Corcuera JI, Etxeberria E, Danyluk MD, Rodrick GE. Metabolomic analysis in food science: a review. Trends Food Sci Tech 2009; 20: 557-566

[79] Mari A, Lyon D, Fragner L, Montoro P, Piacente S, Wienkoop S, Egelhofer $\mathrm{V}$, Weckwerth W. Phytochemical composition of Potentilla anserina L. analyzed by an integrative GC-MS and LC-MS metabolomics platform. Metabolomics 2013; 9: 599-607

[80] Stevigny C, Rolle L, Valentini N, Zeppal G. Optimization of extraction of phenolic content from hazelnut shell using response surface methodology. J Sci Food Agr 2007; 87: 2817-2822

[81] Odabas HI, Koca I. Application of response surface methodology for optimizing the recovery of phenolic compounds from hazelnut skin using different extraction methods. Ind Crop Prod 2016; 91: 114-124

[82] Kornsteiner M, Wagner KH, Elmadfa I. Tocopherols and total phenolics in 10 different nut types. Food Chem 2006; 98: 381-387

[83] Gultekin-Ozguven M, Davarci F, Pasli AA, Demir N, Ozcelik B. Determination of phenolic compounds by ultra high liquid chromatography-tandem mass spectrometry: applications in nuts. LWT-Food Sci Technol 2015; 64: 42-49

[84] Persic M, Mikulic-Petkovsek M, Slatnar A, Solar A, Veberic R. Changes in phenolic profiles of red-colored pellicle walnut and hazelnut kernel during ripening. Food Chem 2018; 252: 349-355

[85] Gokirmak T, Mehlenbacher S, Bassil N. Characterization of European hazelnut (Corylus avellana) cultivars using SSR markers. Genet Resour Crop Ev 2009; 56: 147-172

[86] Sciubba F, Di Cocco ME, Gianferri R, Impellizzeri D, Mannina L, De Salvador FR, Venditti A, Delfini M. Metabolic profile of different Italian cultivars of hazelnut (Corylus avellana) by nuclear magnetic resonance spectroscopy. Nat Prod Res 2014; 28: 1075-1081

[87] Caligiani A, Coisson JD, Travaglia F, Acquotti D, Palla G, Palla L, Arlorio M. Application of ${ }^{1} \mathrm{H}$ NMR for the characterisation and authentication of “Tonda Gentile Trilobata” hazelnuts from Piedmont (Italy). Food Chem 2014; 148: 77-85

[88] Bachmann R, Klockmann S, Haerdter J, Fischer M, Hackl T. ${ }^{1} \mathrm{H}$ NMR spectroscopy for determination of the geographical origin of hazelnuts. J Agric Food Chem 2018; 66: 11873-11879

[89] Napolitano A, Cerulli A, Pizza C, Piacente S. Multi-class polar lipid profiling in fresh and roasted hazelnut (Corylus avellana cultivar "Tonda di Giffoni”) by LC-ESI/LTQOrbitrap/MS/MSn. Food Chem 2018; 269: 125135 